\title{
Field correlator method for the confinement in QCD
}

\author{
Yu. A. Simonov \\ State Research Center, Institute of Theoretical and Experimental Physics, Moscow, 117218 Russia
}

(Received 21 May 2018; revised manuscript received 21 January 2019; published 26 March 2019)

\begin{abstract}
The theory of confinement based on the stochastic field mechanism, known as the field correlator method (FCM) is discussed in detail. Experimental and lattice data have accumulated a vast amount of material on the properties of confinement in QCD. We enumerate all these properties as (1)-(7), and discuss beyond FCM two existing approaches: monopole based dual Ginzburg-Landau (DGL) theory, and GribovZwanziger model, from this point of view. It is shown that the FCM satisfies all required criteria. We also prove its self-consistency; in particular, it is shown that the string tension $\sigma$ is the only scaleful parameter in the theory beyond fermion masses, and $\Lambda_{\mathrm{QCD}}$ is calculated explicitly to the lowest order in terms of $\sigma$. We also formulate physical consequences of confinement, such as string breaking, Regge trajectories, role of confinement in the perturbation theory, chiral symmetry breaking, confinement in the boosted systems etc. It is demonstrated that the FCM is a suitable tool for the solution of these problems.
\end{abstract}

DOI: 10.1103/PhysRevD.99.056012

\section{INTRODUCTION}

The problem of confinement and its internal structure remains an important issue nowadays, while this topic is studied in numerous papers for the last 45 years, starting from the first papers [1-4]. It was generally assumed at first, that the most important role in confinement must play topologically nontrivial configurations, e.g., like magnetic monopoles [2-5] or else other classical solutions: (multi) instantons, dyons, etc. The corresponding effective Lagrangians, establishing the form of dual Abrikosov fluxes [6,7], have soon been found [8,9] and demonstrated possibility of the dual Ginzburg-Landau (DGL) theory. This topic is effectively elaborated on till now, see $[10,11]$ for reviews.

Since the first definition of confinement via the area law of the Wilson loop [1], the lattice analysis of confinement plays the most important role, which allows to define the most important properties of confinement and study this phenomenon quantitatively, see [12]. These studies allowed us to analyze the QCD vacuum configurations and to search for monopolelike degrees of freedom (d.o.f.), as it is done in the Abelian projection method (APM) [13], in the center vortex model [14], and the thick vortex model [15].

Meanwhile another approach to confinement, based on the field correlator method (FCM), was formulated in [16], see [17-19] for reviews, which allowed us to connect

Published by the American Physical Society under the terms of the Creative Commons Attribution 4.0 International license. Further distribution of this work must maintain attribution to the author(s) and the published article's title, journal citation, and DOI. Funded by SCOAP ${ }^{3}$. confinement directly to the (Gaussian) bilocal field correlator $\langle F(x) F(y)\rangle$, created in the QCD vacuum, and therefore sometimes called the stochastic confinement (SC). One of the most crucial tests of this method is the analysis of confinement between sources in different group representations - the so-called Casimir scaling (CS), which was done on the lattice [20-22] and compared to the FCM predictions $[23,24]$. In [22] the agreement was around 5\% for all 8 studied representations, which strongly supported the FCM approach.

At the same time the analysis of CS in the DGL model $[8,25]$ has shown that the $\mathrm{SU}(3)$ Casimir ratios cannot be reproduced for the fixed parameters of the model, i.e., for fixed values of the monopole mass $m_{\chi}$ and dual gauge field mass $m_{B}$, so that each representation requires its own set of masses. Till now this discrepancy has not been resolved and a reasonable modification of the DGL type or any other connected model, satisfying Casimir scaling for SU(3) or $\mathrm{SU}(\mathrm{N})$ groups, has not yet been found, which sets some limits on the presence of DGL configurations in the QCD vacuum.

This analysis can be prolonged to take into account the simple groups $F_{4}, E_{6}$, and $G_{2}$, where linear confinement is present only up to some distance, see [26-32]. Here the aim is to find the connection between the group structure and spatial and Casimir properties of confinement. As a basic point one can use here also the bilocal field correlators of the exceptional groups, which provide Casimir scaling.

One of the most important issues of the CS is the proof of the dominance of the bilocal correlator, which ensures CS, and the estimates of higher correlators, $\langle F F F F\rangle$ etc. This analysis was first done in $[33,34]$, where it was shown that all properties of the correlators and, moreover, the 
quantitative expression for bilocal correlator, i.e., the most part of the confinement dynamics, can be derived from the gluelump Green's functions, which were found analytically in [35] and on the lattice in [36].

In this way the theory of confinement has found its quantitative basis and can be called the FCM of confinement. In this theory the only parameter is the string tension $\sigma$ (which may be expressed via $\Lambda_{\mathrm{QCD}}$ ) and the field correlators are expressed in terms of $\sigma$, which finally yields self-consistent connection $\sigma(\sigma)$ [33]. We consider this fact as the most important property of our approach, which is missed in the DGL type of models, where masses $m_{B}$ and $m_{\chi}$ and their ratio are not introduced till now self-consistently.

All this and additional physical properties, taken from lattice and hadron properties, can be formulated as necessary properties of the QCD confinement mechanism, listed below.

(1) Confinement is linear in the $S U(N)$ field theory for all measured distances, $R \lesssim 1 \mathrm{fm}$ as found on the lattice [12,22], whereas for QCD with $n_{f}>0$ at large distances linear confinement is flattening. Field correlators in QCD are exponentially damped at large distances [37-41], $\langle F F\rangle \sim \frac{c}{x^{4}}+d \exp (-\mu x)$.

(2) Casimir scaling is found for all charge representations of $S U(3)$ with $\mathrm{O}(5 \%)$ accuracy in the range up to $1 \mathrm{fm}[22]$.

(3) Flux tubes are observed between the charges, with the radius, which is slowly changing with distance between charges, see [12,42]. A circular colormagnetic current is observed around flux tubes, asymptotically satisfying the dual London equation $[17,19]$. The excited flux tubes have the specific hybrid type spectrum [20]. The $3 q$ and $3 g$ systems have the string configuration of the string junction and triangular type, respectively [12].

(4) When going from static charges to finite mass fermions, one discovers the necessity of the scalar property of confinement, since otherwise the vector confinement does not ensure $q \bar{q}$ bound states $[43,44]$.

(5) Since we have the only scale in $\mathrm{QCD}, \sigma$ or $\Lambda_{\mathrm{QCD}}$, which defines all quantities (in addition to quark masses), the confinement interaction should be expressed via $\Lambda_{\mathrm{QCD}} \sim \sqrt{\sigma}$ as the only scale parameter.

(6) The confinement theory should explain the interaction between Wilson loops, observed on the lattice [45], in good agreement with FCM [46], and in particular, the visible lack of interaction between the Coulomb-like fluxes and colored flux tubes, which ensures the nonscreening of the Coulomb interaction at large distances, observed on the lattice $[12,22]$ and in the hadron spectrum [47], and explained in [48] within the FCM.

(7) The confinement theory should be applied to all known examples of field theory, e.g., it should yield no confinement for QED, linear confinement for all groups $S U(N), N \geq 2$. It can be also applied to other simple groups, like $G_{2}, F_{4}, H_{6}$, where linear confinement with $C S$ is present in the region $r<r_{\max }$ [26-32,49].

Below we apply these properties as criteria to three types of confinement mechanisms, the FCM, the DGL theory, and the Gribov-Zwanziger [50] approach. We also discuss the center vortex models $[14,15]$ and their possible connection to the FCM.

As we demonstrate below, using the concrete gluelump structure of the field correlators developed in $[33,34]$, the FCM satisfies all criteria. This has allowed to calculate the confinement interaction at all distances, ensuring linear confinement for $r>\lambda \sim 0.1 \mathrm{fm}$, where $\lambda$ is the inverse mass of the lowest gluelump, $M_{\mathrm{Glp}} \approx 2 \mathrm{GeV}$, calculated via string tension $\sigma$. This theory was applied to the structure of flux tubes, originally in [17,19] and recently in [48]. Surprisingly, our flux tubes confirm all the structure, observed on the lattice $([12,22,42])$, implying, e.g., also the asymptotic validity of dual London equation. This means, that the main mechanism of dual magnetic vacuum, providing mass of propagating gluons, is present in our "microscopic" Gaussian-Gluelump approach, resembling in this respect the macroscopic DGL approach.

It is remarkable that starting directly from simplest (Gaussian) field correlators and not assuming any of DGL configurations, one arrives on the microscopic level (i.e., on distances $x \geq \lambda \cong 0.2 \mathrm{fm}$ ) at the field and current distributions specific for the macroscopic DGL equations.

Indeed, as we shall show below, for the most general form of field correlators one obtains the circular color magnetic currents $\mathbf{k}_{D}$ around flux tubes, which satisfy asymptotically the dual London's equation $\operatorname{rot} \mathbf{k}_{D}=$ $\lambda^{-2} \mathbf{E}_{D}$ and there emerges picture of dual superconducting fluxes around the dual Abrikosov string. In all this picture no magnetic monopole d.o.f. are needed, the only microscopic reason of confinement is the presence of the scalar $D(z)$ in the vacuum correlator $\left\langle F_{\mu \nu}(x) \Phi F_{\lambda \sigma}(y)\right\rangle \sim$ $(\delta \delta-\delta \delta) D(x-y)+\ldots$.

The presence of such term in the QCD vacuum with or without quarks is proved by numerous lattice calculations [37-41], and in FCM it is calculated in a self-consistent way. Then one may ask oneself: why at all one should search for magnetic-monopolelike d.o.f. in the QCD configurations? Why one needs any topological configurations, since a simple scalar term $D(z)$ in the microscopic correlator $\langle F F\rangle$ already ensures the macroscopic dual superconducting picture?

Or in other words: what additional features of confinement are provided by the DGL type of theory? As we shall see in the next chapters, one still has no explicit answer to this question in $\mathrm{QCD}$, or $\mathrm{SU}(\mathrm{N})$ theories, where the FCM alone is sufficient to explain all known details of confinement till now, but in more complicated theories like $G_{2}, F_{4}, H_{6}$, one may need other instruments, like the DGL or center vortex model. 
In what follows we shall shortly derive and discuss basic equations of our method (to be referred to as FCM), demonstrate how it satisfies the conditions (1)-(7), and find the points, where other approaches fail.

The plan of the paper is as follows. In Sec. II we give the basics of the FCM and in Sec. III discuss the properties (1)-(7) within FCM. In Sec. IV the DGL approach is discussed with respect to the same properties. In Sec. V the approach of center vortex model (CVM) is shortly exposed and compared to the FCM approach. In Sec. VI the necessary scalar property of confinement is proved for light quarks. In Sec. VII we discuss five features of the QCD dynamics, which are connected with confinement, and demonstrate how the FCM is incorporated in resolving: the IR renormalon problem, chiral symmetry breaking, string breaking, confinement in boosted systems, spin-dependent interaction etc. The concluding section gives the summary of results and discussion of possible development.

\section{BASICS OF THE FIELD CORRELATOR APPROACH}

One starts with the expression of the Wilson loop [1], which can be also rewritten in terms of the field strength operators $F_{\mu \nu}$, using the non-Abelian Stokes theorem [51] for the minimal surface $S_{\min }$ inside the contour $C$

$$
\begin{aligned}
W(C) & =\frac{1}{N_{c}}\left\langle\operatorname{tr} P \exp \left(i g \int_{C} d z_{\mu} A_{\mu}(z)\right)\right\rangle \\
& =\frac{1}{N_{c}}\left\langle\operatorname{tr} P \exp \left(i g \int_{S_{\min }} d \sigma_{\mu \nu} F_{\mu \nu}\right)\right\rangle .
\end{aligned}
$$

One can apply to (1) the operator cluster expansion [52], which allows us to expand in the exponent the connected terms, producing connected correlators $\langle\langle\rangle\rangle$.

Before doing this one should define the gauge-covariant quantity, $\hat{F}(x)=\Phi(X, x) F(x) \Phi(x, X)$, where $\Phi(x, y)=$ $P \exp i g \int_{y}^{x} A_{\mu}(z) d z_{\mu}$ is a parallel transporter (pt), and we have chosen $x=X$ as an arbitrary common point to make all construction gauge invariant. Thus each $\hat{F}(x)$ is connected to $X$ by a pair of pt. Further on, in doing the vacuum averaging of products $\langle\hat{F}(1) \hat{F}(2) \ldots \hat{F}(n)\rangle$, one is exploiting the minimal action principle, which ensures that the main contribution to the average is given by the configuration where all points $1,2, \ldots n$ are connected by pt's of minimal length, so that $\langle\hat{F}(1) \hat{F}(2)=\langle\Phi(2,1) F(1) \Phi(1,2) F(2)\rangle$ and so on. As a result the vacuum averaging due to [52] yields

$$
\begin{aligned}
W(C)= & \frac{1}{N_{c}} \operatorname{tr} \exp \left[-\frac{g^{2}}{2} \int d \sigma_{\mu \nu} d \sigma_{\lambda \rho}\left\langle\left\langle F_{\mu \nu} F_{\lambda \rho}\right\rangle\right\rangle\right. \\
& +\frac{g^{4}}{4 !} \int d \sigma(1) d \sigma(2) d \sigma(3) d \sigma(4) \\
& \left.\times\langle\langle\hat{F}(1) \hat{F}(2) \hat{F}(3) \hat{F}(4)\rangle\rangle+O\left(g^{6}\right)\right] .
\end{aligned}
$$

We shall keep the first term in the exponent $O\left(g^{2}\right)$ as the basic approximation and later estimate other terms, following the discussion in $[18,23,24]$. From $[19,51,52]$ one can deduce that one can organize the connected clusters $\langle\langle F(1) \ldots F(n)\rangle\rangle$ in such a way, that all points $1, \ldots n$ are connected by interaction, averaged in the vacuum averaging process. Assuming the correlation length $\lambda$ for this interaction, one obtains the estimate

$$
\begin{aligned}
I_{n} & \equiv \iint\langle\langle F(1) \ldots F(n)\rangle\rangle d \sigma(1) \ldots d \sigma(n) \\
& \approx f R T_{4}\left(\lambda^{2} f\right)^{n-1},
\end{aligned}
$$

where $f$ is the order of magnitude estimate of the operator $F$.

The basic point of the FCM is the expression for the vacuum field correlator, which in the color-electric case is [16]

$$
\begin{aligned}
& g^{2} D_{i 4 k 4}^{(2)}(x-y) \\
& \quad \equiv \frac{g^{2}}{N_{c}}\left\langle\operatorname{tr}_{f}\left(F_{i 4}(x) \Phi(x, y) F_{k 4}(y) \Phi(y, x)\right\rangle\right. \\
& \quad=\left(\delta_{i k}\right) D^{E}(x-y)+\frac{1}{2}\left(\frac{\partial}{\partial x_{i}}\left[h_{k}+\text { perm }\right]\right) D_{1}^{E}(x-y), \\
& h_{\lambda}=x_{\lambda}-y_{\lambda}, \quad(x-y)^{2}=\sum_{\lambda=1}^{4}\left(x_{\lambda}-y_{\lambda}\right)^{2} .
\end{aligned}
$$

Insertion of (4) into (2) yields the area law of the Wilson loop

$$
W(C)=\exp \left(-\sigma R T_{4}\right), \quad \sigma=\frac{1}{2} \int d^{2} z D^{E}(z) .
$$

Comparing (4) with (3), one can see that the estimate holds

$$
\sigma \approx f^{2} \lambda^{2}, \quad I_{n} \sim \frac{R T_{4}}{\lambda^{2}}\left(\sigma \lambda^{2}\right)^{n / 2}, \quad \frac{I_{4}}{I_{2}} \sim \sigma \lambda^{2}
$$

In the Appendix A we estimate in detail the quartic correlator, supporting validity of Eq. (6).

We now turn to the calculation of static potentials generated by $D^{E}, D_{1}^{E}$.

We start with the Wilson loop, $W(C)=$ $\left.\exp \left(-\frac{g^{2}}{2} \int d \sigma \int d \sigma\langle F F\rangle\right)=\exp \left(-\int\left(V_{D}^{R}\right)+V_{1}(R)\right) d t_{4}\right)$ and consider an interval $\Delta t_{4}>\lambda$ in both integrals $\int d t_{4}$ and $\int d \sigma(u) \int d \sigma(v)$, which yields (see Fig. 1), $d \sigma(u)=d u_{4} d u_{1}$. 


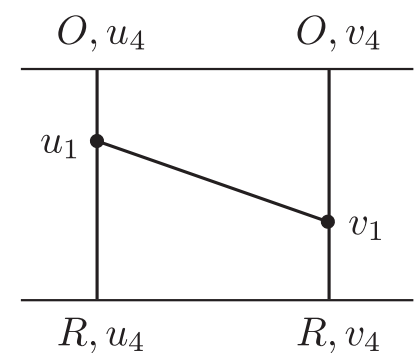

FIG. 1. Calculation of the static potentials $V_{D}, V_{1}$ from field correlators.

$$
\begin{aligned}
& V_{D}(R) \Delta t_{4} \\
& =2 \int_{0}^{R} d u_{1} \int_{0}^{\Delta t_{4}} d u_{4} \int_{0}^{R} d v_{1} \int_{0}^{\Delta t_{4}} d v_{4} D^{E}(u-v) \\
& =2 \int d \frac{u_{1}+v_{1}}{2} \int d\left(u_{1}-v_{1}\right) \int d \frac{u_{4}+v_{4}}{2} \\
& \quad \times \int d\left(u_{4}-v_{4}\right) D^{E}(u-v) \\
& =\Delta t_{4} 2 \int_{0}^{R}\left(R-w_{1}\right) d w_{1} \int_{0}^{\Delta t_{4}} d w_{4} D^{E}\left(\sqrt{w_{1}^{2}+w_{4}^{2}}\right) .
\end{aligned}
$$

As a result for $\Delta t_{4} \gg \lambda$, one obtains the static potential $V_{D}(R)$ for the fundamental charges

$$
\begin{aligned}
V_{D}(R) & =2 \int_{0}^{R}\left(R-w_{1}\right) d w_{1} \int_{0}^{\infty} d w_{4} D^{E}\left(\sqrt{w_{1}^{2}+w_{4}^{2}}\right) \\
& =V_{\text {conf }}(R)+V_{D}^{\text {sat }}(R),
\end{aligned}
$$

where $V_{D}^{\text {sat }}(R)$ is negative and saturates at large $R$.

For the charge representation $D$ the Gaussian correlator $D^{E}$ defines the interaction between static charges in the representation $D=3,8,6, \ldots$

$V_{D}(R)=C_{D} \int_{0}^{R}\left(R-w_{1}\right) d w_{1} \int_{0}^{\infty} d w_{4} D^{E}\left(\sqrt{w_{1}^{2}+w_{4}^{2}}\right)$,

where $C_{D}=2 \frac{C_{2}(D)}{C_{2}(f)}$, and $C_{2}(D)$ is the quadratic Casimir coefficient for the representation $D[16,18]$.

In a similar way one obtains static potential $V_{1}(R)$, generated by $D_{1}^{E}(z),[17,18]$

$$
V_{1}(R)=\int_{0}^{R} w_{1} d w_{1} \int_{0}^{\infty} d w_{4} D_{1}^{E}\left(\sqrt{w_{1}^{2}+w_{4}^{2}}\right)
$$

As it is shown in Appendix B, after regularization one arrives at the final form of $V_{1}(R)$ in (10)

$$
V_{1}(R)=-C_{2} \frac{\alpha_{s}}{R}+V_{1}^{\mathrm{sat}}(R) .
$$

Since all components of $V_{D}, V_{1}$ are proportional to the quadratic field correlator, the potential for any representation $D=f, \operatorname{adj}, \ldots$ are proportional to the coefficient $C_{2}(D)$, where $C_{2}(D)$ is the Casimir factor.

The most important step was done in $[33,34]$, where $D_{1}^{E}$ and $D^{E}$ were expressed via the one- and two-gluon gluelump Green's function, as shown in Figs. 2 and 3, respectively.

Indeed, writing $F_{\mu \nu}$ in (4) as $F_{\mu \nu}=\partial_{\mu} A_{\nu}-\partial_{\nu} A_{\mu}+$ $i g\left[A_{\mu}, A_{\nu}\right]$, one obtains for $g^{2} D_{\mu \nu, \lambda \sigma}^{(2)}$ in (4) the derivative terms, denoted as $D_{1, \mu \nu \lambda \sigma}$ and $O\left(g^{4}\right)$ terms proportional to $\left\langle\left[A_{\mu}, A_{\nu}\right] \Phi(x, y)\left[A_{\lambda}, A_{\sigma}\right]\right\rangle \equiv G^{(2 g)}$. It is clear, that $D_{1 \mu \nu \lambda \sigma}$ contain the term $\left\langle A_{\mu}(x) \Phi(x, y) A_{\lambda}(y)\right\rangle=G^{(1 g)}(x, y)$ which is the one-gluon-gluelump Green's function, while $G^{(2 g)}$ is the two-gluon gluelump Green's function, calculated in $[35,36]$. A more detailed derivation is discussed in Appendix B. As a result one can associate $D_{1}^{E}$ with the derivative terms in correlator $\langle F \Phi F\rangle$, namely,

$$
\begin{aligned}
D_{1 \mu \nu, \lambda \sigma}(x, y)= & \frac{g^{2}}{2 N_{c}^{2}}\left\{\frac{\partial}{\partial x_{\mu}} \frac{\partial}{\partial y_{\lambda}}\left\langle\operatorname{tr}_{a} A_{\nu}(x) \Phi(x, y) A_{\sigma}(y)\right\rangle\right. \\
& + \text { perm }\},
\end{aligned}
$$

and $D_{1}$ is expressed via the one-gluon gluelump Green's function with the asymptotics found in $[33,34]$

$D_{1}^{E}(x)=-\frac{2 g^{2}}{N_{c}^{2}} \frac{d G^{(1 g)}(x)}{d x^{2}}, \quad D_{1}^{E}(x) \approx \frac{A_{1}}{|x|} e^{-M_{1}|x|}$,

where $A_{1}=2 C_{2} \alpha_{s} \sigma_{\text {adj }} M_{1}$.

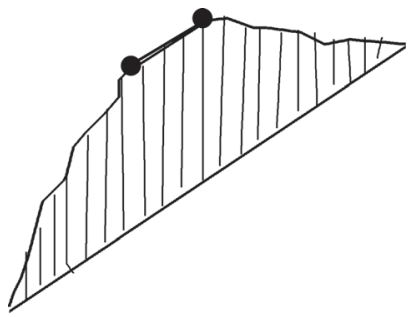

FIG. 2. The one-gluon gluelump Green's function.

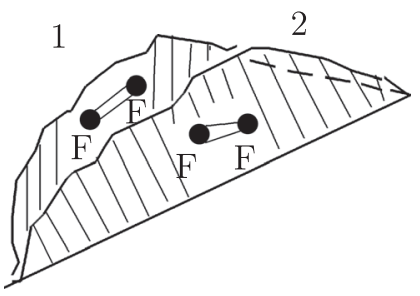

FIG. 3. The two-gluon gluelump Green's function. 
One can see that $g g \Phi$ is the bound state of two gluons with a static gluon from $\Phi(x, y)$, so that in the transverse plane the $g g \Phi$ configuration looks like a triangle, where straight lines of its sides represent confining strings between adjoined charges. A similar consideration for the $O\left(g^{4}\right)$ terms yields $D^{E}(x-y)$.

$$
\begin{aligned}
D^{E}(x-y) & =\frac{g^{4}\left(N_{c}^{2}-1\right)}{2} G^{(2 g)}(x, y) \\
& =g^{4} N_{c} C_{2}(f) G^{(2 g)}(x, y) .
\end{aligned}
$$

The spectrum of $G^{(2 g)}$ was found both analytically [35] and on the lattice [36], and in [33,34] the asymptotics was found as

$$
G^{(2 g)}(x) \approx 0.108 \sigma_{f}^{2} e^{-M_{0}^{(2 g)}|x|}, \quad x \gtrsim\left(M_{0}^{(2 g)}\right)^{-1}
$$

while $M_{0}^{(2 g)} \approx 2 \mathrm{GeV},\left(M_{0}^{(2 g)}\right)^{-1} \equiv \lambda_{0}=0.1 \mathrm{fm}$. The mixing of $M^{(2 g)}$ and $M^{(1 g)}$ and the account of color Coulomb interaction imply the lowering of $M^{(2 g)}$, and hence the increasing of $\lambda, \lambda \lesssim 0.2 \mathrm{fm}$. Note here, that $M_{0}^{(2 g)}$ is expressed via $\sigma$, namely, according to $[35,36]$.

$$
M_{0}^{(2 g)} \cong(4-4.5) \sqrt{\sigma_{f}}
$$

and it is the large ratio $4 \gg 1$, that ensures the small ratio of $\frac{I_{n}}{I_{2}}$ in (6), e.g.,

$$
\frac{I_{4}}{I_{2}} \sim \frac{\sigma}{(4.5 \sqrt{\sigma})^{2}} \sim \frac{1}{20} .
$$

This result will be basic for the properties (1) and (2), listed in the Introduction.

One can compare this result for $\lambda$ with direct lattice measurements of field correlators [37-41], which yield $\lambda \approx 0.2 \mathrm{fm}$.

We are now coming to the most important property of the FCM mechanism of confinement-the scale selfconsistency. Indeed, in FCM (and in QCD with massless quarks in general) one has the only nonperturbative scale, which defines $99 \%$ of mass in the visible part of the universe. It can be chosen as a scale since confinement and $\sigma$ explain the nucleon masses. Now from the definition of $\sigma$ (to the lowest order in $\alpha_{s}$ ) $\sigma_{f}=\frac{1}{2} \int D^{E}(x) d^{2} x$, and the asymptotic expressions (14), (15) for $D^{E}(x)$ one obtains the self-consistency condition

$$
\sigma_{f} \lesssim \pi \lambda^{2} \cdot 0.0108 \cdot 8 \pi^{2} \cdot \alpha_{s}^{2}\left(N_{c}^{2}-1\right) \sigma_{f}^{2} .
$$

(The sign $<$ is due to overestimating $D^{E}(x)$ using asymptotics (15), since $D^{E}$ is smaller for $x \rightarrow 0$, and has a minimum at $x=0$, as shown in Appendix C). Here $\lambda=$ $1 / M$ and we can associate the momentum scale of $\alpha_{s}$ with the gluelump mass $M \cong 2 \mathrm{GeV} \cong 5 \sqrt{\sigma}$, and from (18) one finds the dependence $\alpha_{s}(M)$

$$
\begin{aligned}
\alpha_{s}^{2}(M) \equiv & \left(\alpha_{s}^{*}\right)^{2}=\frac{M^{2} \cdot 0.037}{\sigma_{f}\left(N_{c}^{2}-1\right)} \cong 0.104 \\
& \left(N_{c}=3, \alpha_{s}^{*}=0.322, M=2 \mathrm{GeV}\right)
\end{aligned}
$$

on the other hand one can use the one-loop approximation for $\alpha_{s}(M)$ with the IR correction found earlier (see Appendix $\mathrm{C}$ for details).

$\alpha_{S}(M)=\frac{4 \pi}{\beta_{0} \ln \left(\frac{M^{2}+M_{B}^{2}}{\Lambda^{2}}\right)}, \quad M_{B} \cong 2 \pi \sigma_{f} \approx 1 \mathrm{GeV}$

which yields [taking into account the sign $<$ in (18)] for $N_{c}=3$

$$
\Lambda_{\sigma} \gtrsim \sqrt{M^{2}+M_{B}^{2}} \exp \left(-\frac{4 \pi}{\beta_{0} \alpha_{s}^{*}}\right) \cong 270 \mathrm{MeV} .
$$

As one can see in (21) the $N_{c}$ dependence in $\alpha_{s}^{*} \beta_{0}$ is compensated at large $N_{c}$, and the limiting value of $\Lambda_{\sigma}\left(N_{c}=\infty\right)$ is equal to $340 \mathrm{MeV}$, and as discussed in Appendix C, Eq. (C5) should be divided by 1.3 to compare favorably with $\Lambda_{\mathrm{QCD}}^{\overline{\mathrm{MS}}}$. The resulting values of $\alpha_{s}(2 \mathrm{GeV})$ and $\Lambda_{\mathrm{QCD}}^{\overline{\mathrm{MS}}}$ are well within the PDG limits.

In this way we have expressed to the lowest order the $\Lambda_{\mathrm{QCD}}$ via the string tension, which can be now considered as the only scale constant in QCD beyond the fermion masses.

We turn now to the phenomenon of flux tubes and their internal structure. It is a widespread notion that flux tubes are a necessary and unique result of the DGL theory, producing a dual magnetic flux in the medium filled by the Higgs-like monopole condensate. However one obtains the similar picture of a flux tube directly from the quadratic field correlators and without any additional parameters except $\sigma$ and $\lambda=\frac{1}{c \sqrt{\sigma}}, c \approx 4$.

Indeed, following [19,48], one can measure the field $F_{\mu \nu}$ produced in the contour $C$, as shown in Fig. 4 (the so-called connected probe) and write

$$
F_{\mu \nu}(x)=\int_{S} d \sigma_{\alpha \beta}(y) g^{2} D_{\alpha \beta \mu \nu}^{(2)}(x-y),
$$

where $D^{(2)}$ is given in (4). Writing $D^{E}(z)$ for simplicity as

$$
D^{E}(z)=\frac{\sigma}{\pi \lambda^{2}} \exp \left(-\frac{|z|}{\lambda}\right),
$$

[which satisfies both (15) with $\alpha_{s} \approx 0.2$ and (5)], the mixing of $M^{(2 g)}$ and $M^{(1 g)}$ and the account of color Coulomb interaction imply the lowering of $M^{(2 g)}$ and increasing of 


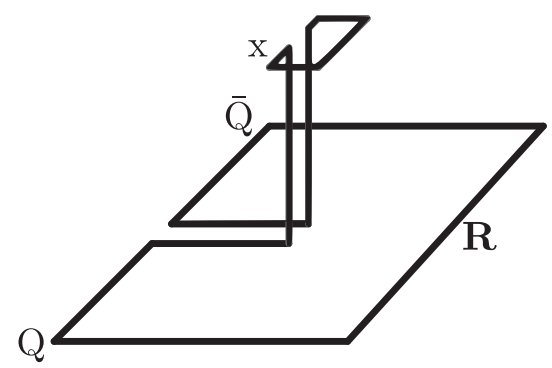

FIG. 4. The connected probe for measuring color field in the flux tube.

$\lambda=\frac{1}{M^{(2 g)}}$. In all applications to flux tubes it is convenient to choose $\lambda$ around the value of $\lambda=0.2 \mathrm{fm}$. From (22) one obtains the color-electric field in the flux tube $[19,48]$

$$
\mathbf{E}^{D}=\mathbf{n} \frac{2 \sigma}{\pi} \int_{0}^{R / \lambda} d u\left|u \mathbf{n}-\frac{\mathbf{r}}{\lambda}\right| K_{1}\left(\left|u \mathbf{n}-\frac{\mathbf{r}}{\lambda}\right|\right)
$$

where $\mathbf{n}$ is along the flux tube. In a similar way one defines the magnetic current $\mathbf{k}^{D}=\operatorname{rot} \mathbf{E}^{D}$, and at the midpoint between charges and at distance $r_{\perp}$ from the axis it is equal to

$$
\mathbf{k}_{D}^{2}\left(r_{\perp}\right)=\frac{4 \sigma^{2} r^{2}}{\pi^{2} \lambda^{4}}\left(\int_{-\frac{R}{2 \lambda}}^{\frac{R}{2 \lambda}} d x K_{0}\left(\sqrt{x^{2}+\frac{r_{\perp}^{2}}{\lambda^{2}}}\right)\right) .
$$

As was shown in [19] the dual London equation: $\operatorname{rot} \mathbf{k}=$ $\lambda^{-2} \mathbf{E}$ is satisfied by (24), (25) asymptotically at $r_{\perp} \rightarrow \infty$, see Fig. 5.

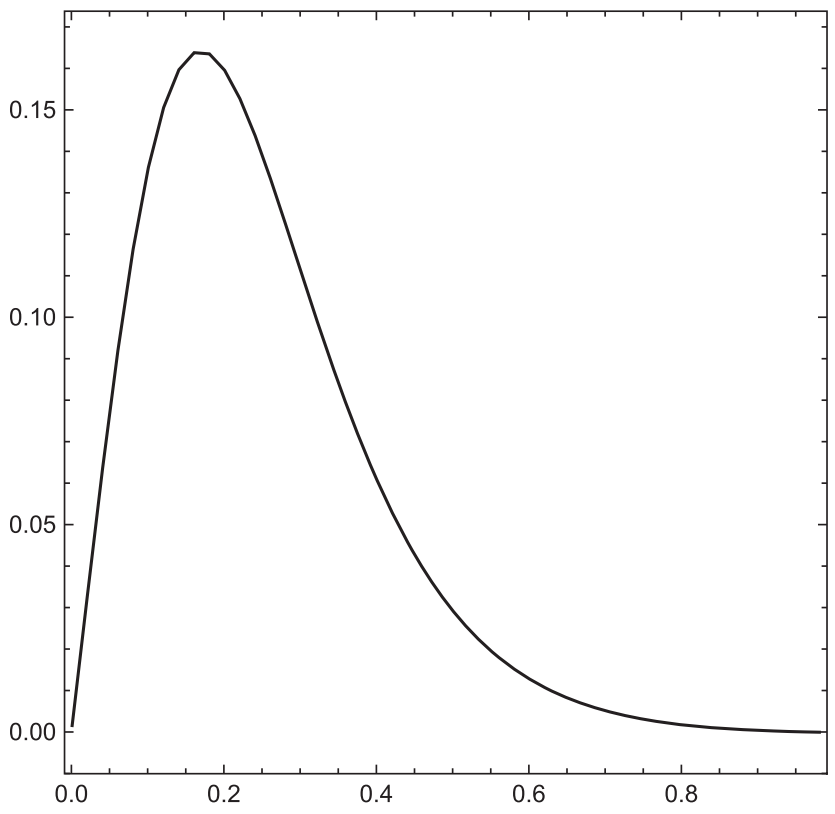

FIG. 5. The transverse radius dependence of the CM current.
To complete this picture one also calculates color-electric fields due to correlator $D_{1}^{E}$, as it is done in the Appendix B, with the resulting field $\mathbf{E}_{1}$, depending on the same parameter $\lambda$. As a result one obtains the profiles of the flux tube for different distances $R$ between charges, shown in Figs. 6-9.

One can see in Figs. 6-9 a rather stable profile, only weakly depending on $R$, in good agreement with lattice data [42].

As was shown above, at the basis of FCM is the property of the Wilson loop, and hence one can immediately derive the effects of confinement, writing any amplitude in terms of Wilson loops. Above we have considered only the simplest case, when all interaction inside $W(C)$ is of $n p$ character. In the Appendix B we have shown that the correlator $D_{1}^{E}$ generates the instantaneous Coulomb interaction $V_{c}(r)$ plus vectorlike interaction $V_{1}^{E}$, entering the Polyakov loop, $L_{f}=\exp \left(-\frac{V_{1}^{(E)}(\infty)}{2 T}\right)$.

In this way the color Coulomb and confinement interaction enter additively the total instantaneous potential, as it is supported by lattice data $[12,21,22]$.

However, one should consider these "dynamical" valence gluons in the confining film. In this case the main point is, how it interacts with the confining film and whether it produces the screening effect in the gluonexchange interaction. This point was studied in [47], where it was shown that the resulting screening is small, with $\mu_{\text {scr }} \lesssim \mathrm{GeV}$ for light quarks and screening is not seen in heavy quarkonia up to distances $\sim 1.2 \mathrm{fm}$. The situation,

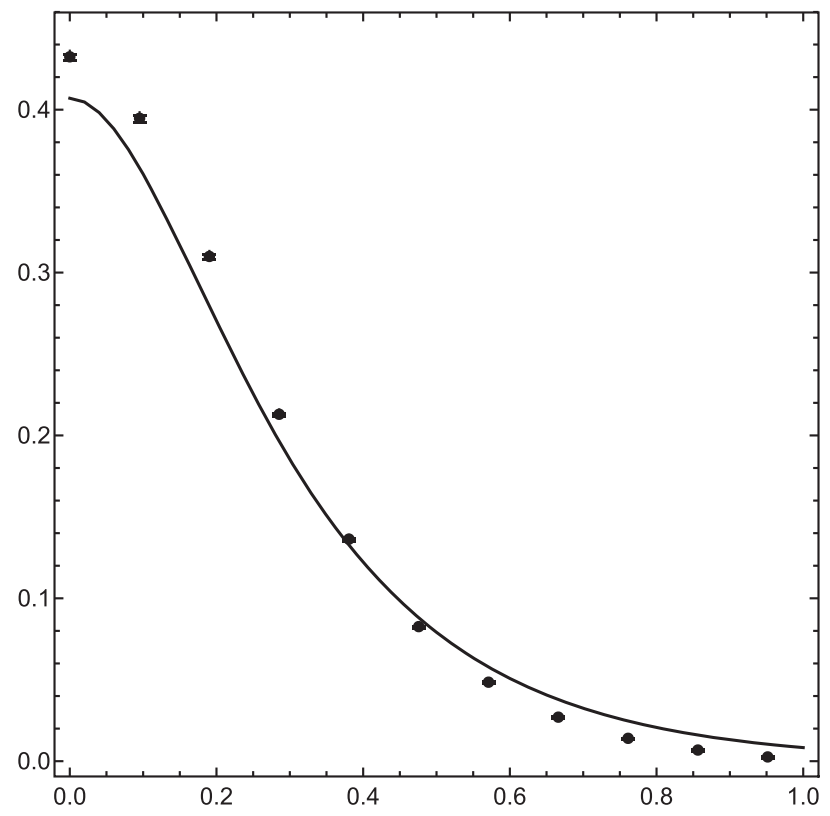

FIG. 6. $E_{3}=E_{3}\left(r_{\perp}, R=0.76 \mathrm{fm}\right)$. The transverse radius dependence of the $\mathrm{CE}$ field strength for the fixed flux tube length $R=0.76 \mathrm{fm}$. The dots with error bars are from the lattice measurements in [42]. 


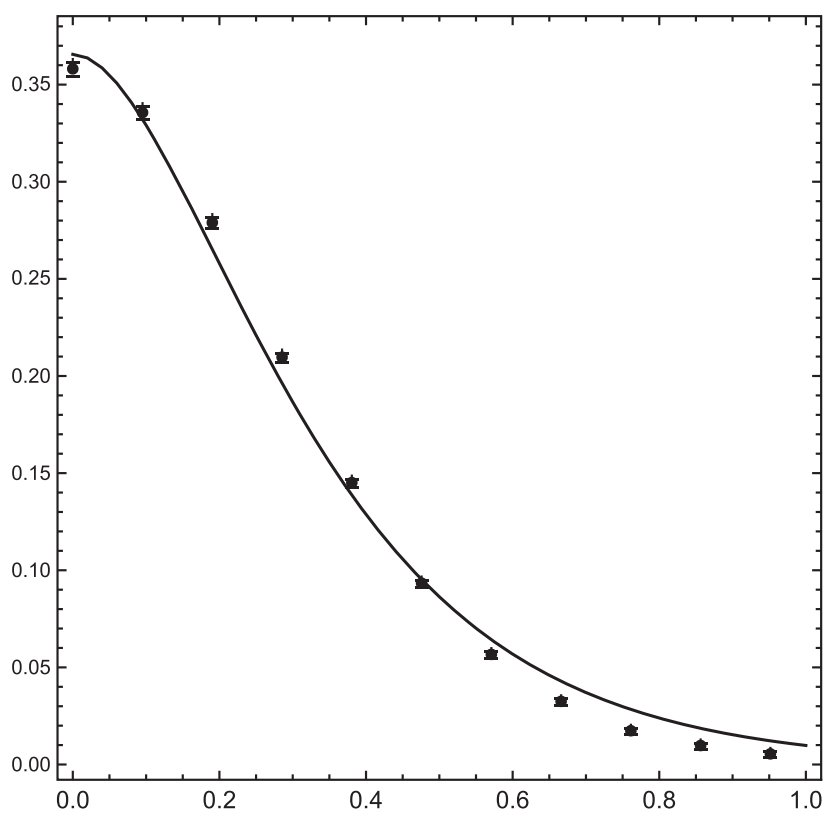

FIG. 7. $E_{3}=E_{3}\left(r_{\perp}, R=0.95 \mathrm{fm}\right)$. The transverse radius dependence of the $\mathrm{CE}$ field strength for the fixed flux tube length $R=0.95 \mathrm{fm}$. The dots with error bars are from the lattice measurements in [42].

when gluon exchange is considered within the confining film, is shown in Fig. 10, and in [48] it was explained why the screening is strongly damped.

The principle of the minimal area for the surface of the Wilson loop operates also for the more complicated objects,

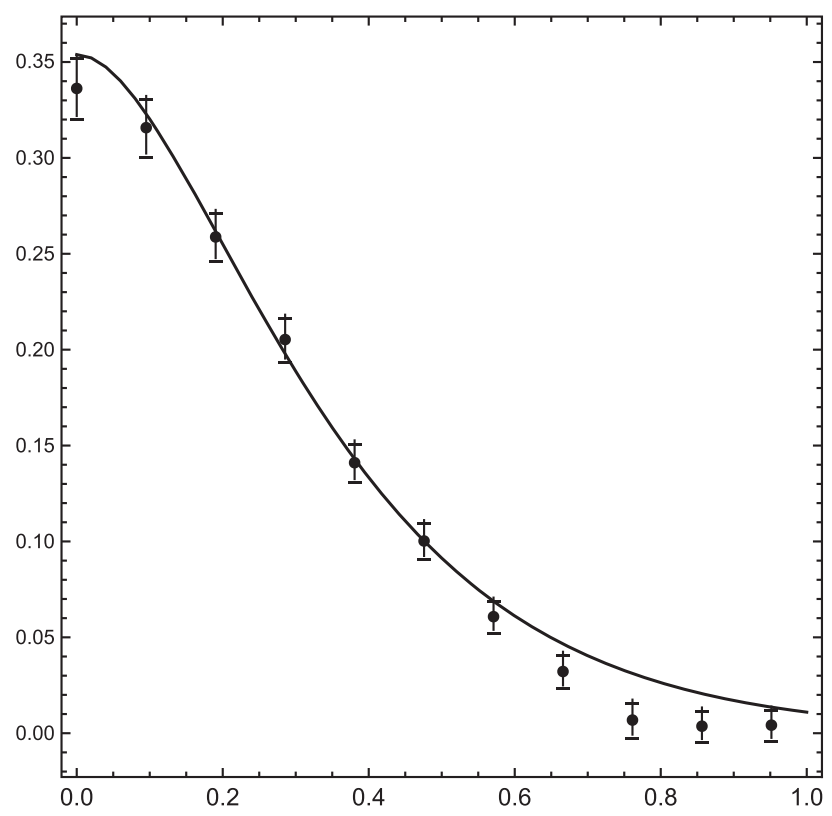

FIG. 8. $E_{3}=E_{3}\left(r_{\perp}, R=1.14 \mathrm{fm}\right)$. The transverse radius dependence of the $\mathrm{CE}$ field strength for the fixed flux tube length $R=1.14 \mathrm{fm}$. The dots with error bars are from the lattice measurements in [42].

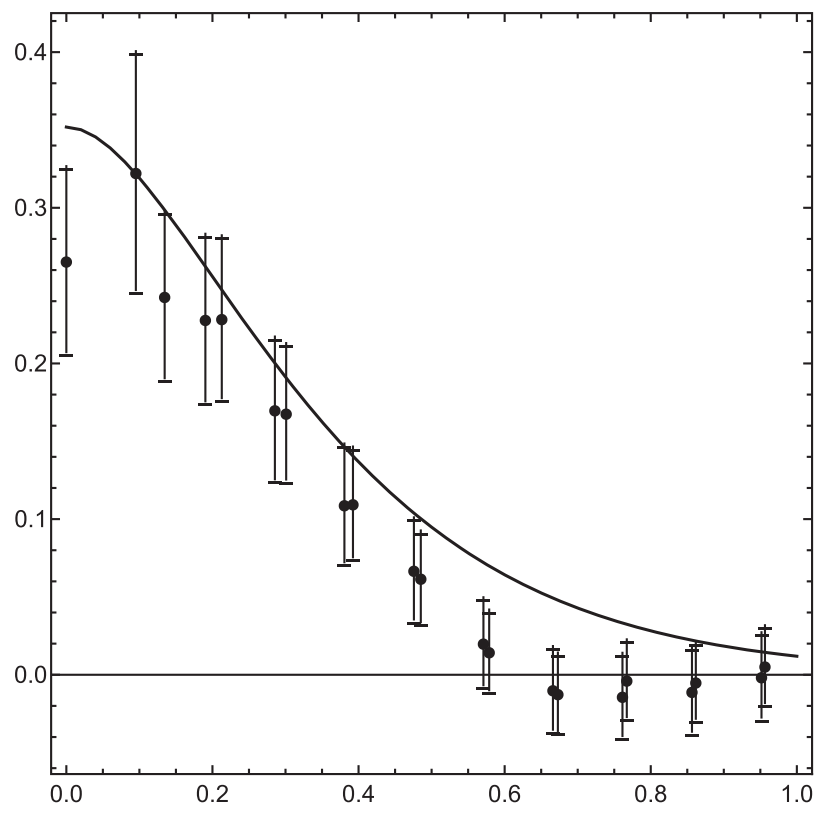

FIG. 9. $E_{3}=E_{3}\left(r_{\perp}, R=1.33 \mathrm{fm}\right)$. The transverse radius dependence of the $\mathrm{CE}$ field strength for the fixed flux tube length $R=1.33 \mathrm{fm}$. The dots with error bars are from the lattice measurements in [39].

like $3 \mathrm{q}$ baryons and $3 \mathrm{~g}$ glueballs, which accordingly have the Wilson loops based on string junction and triangle forms, shown in Fig. 11, see [19] for details and the full theory of 3q baryons in [53].

Finally we touch on the important point of excited QCD strings, which can be treated in the lattice measurements of hybrid states [54], lattice measurements of flux tube excitations [55], and finally in the FCM theory [56]. In the last case the FCM theory predicts excitations of the QCD string in the form of an additional gluon, "sitting on the string," i.e., in the form of local excitation leading to the vibration of the string, with an explicit probability amplitude for the space-time position of the gluon, while in the standard string theory one considers the string as a whole.

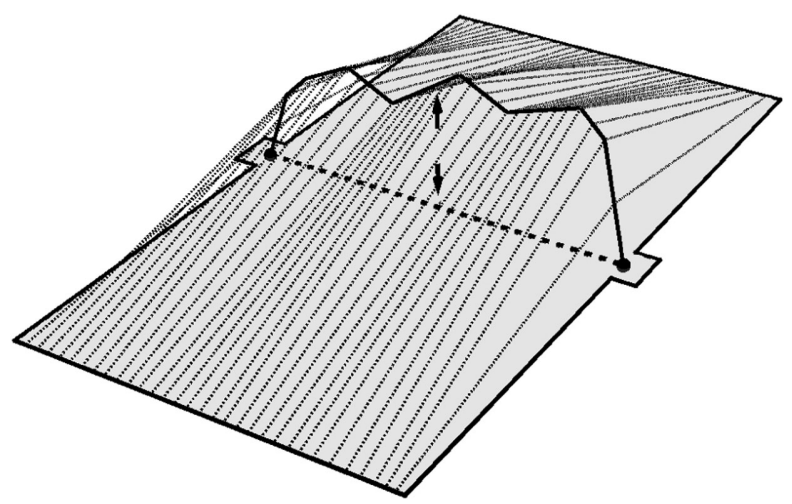

FIG. 10. The minimal area surface for the gluon exchange interaction. 


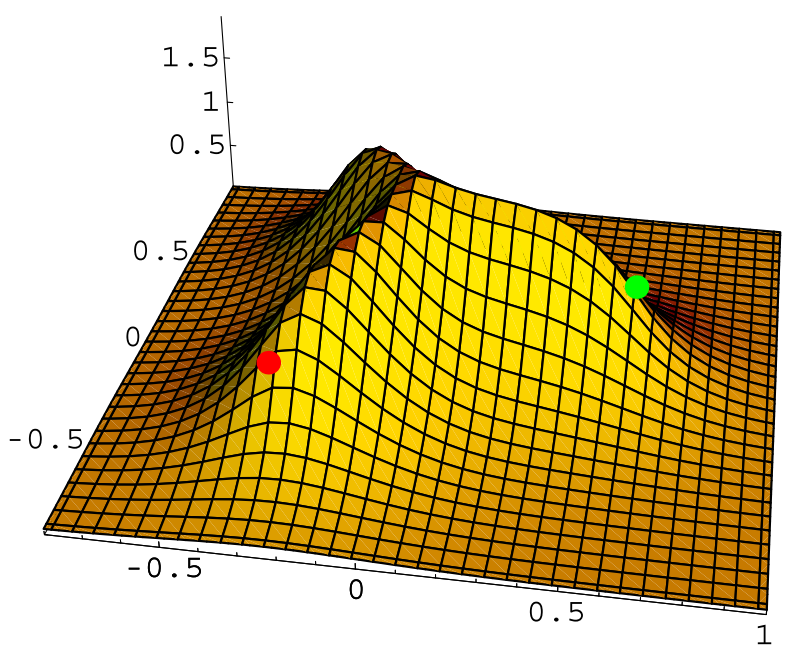

FIG. 11. A distribution of the field $\vec{E}^{(B)}$ in $\mathrm{GeV} / \mathrm{fm}$ with the only correlator $D$ contribution considered in the quark plane for equilateral triangle with the side $1 \mathrm{fm}$. Coordinates are given in $\mathrm{fm}$, positions of quarks are marked by points.

From this point of view the analysis and comparison of the results of [54-56] is of vital importance.

\section{THE FCM THEOR VS PROPERTIES (1)-(7)}

1 . We start with the property (1), stating the observation of the linear confinement in the whole measured region, $0.1 \mathrm{fm}<R<1 \mathrm{fm},[12,21,22]$. As it is clear from (9), (14), (15), taking into account that $D^{E}(u, v)=$ $D^{E}\left(z=\sqrt{u^{2}+v^{2}}\right)$ is essentially nonzero only in the region $r \lesssim \lambda \approx 0.2 \mathrm{fm}$, that the potential $V_{D}(r)$ has a linear behavior for all $r \gtrsim \lambda$ in agreement with all lattice data [12,21,22] and the results in quarkonium structure [57]. This refers to all groups $S U\left(N_{c}\right), N_{c} \geq 2$, however, one should have in mind that in the higher $O\left(g^{n}\right), n \geq 4$ orders or in the nonperturbative $(n p)$ string breaking mechanism the adjoint string may break. This phenomenon of string breaking into two gluelumps brings in the flattening of the confining potential for adjoint and higher $D$ charges, which starts at larger values of $r r \gtrsim r_{\max }$ because of larger gluelump mass. Indeed, since the adjoint string breaks into two gluelumps with mass $\sim 2 \mathrm{GeV}$, one can estimate $r_{\max } \sim \frac{2 M^{(2 g)}}{\sigma_{\text {adj }}} \approx \frac{4 \mathrm{GeV}}{0.189} \mathrm{GeV}^{2} \cong 2 \mathrm{fm}$. A similar situation can occur in the $G_{2}$ group theory, and other special groups [26-32,49].

In FCM the behavior of field correlator clearly agrees with that found on the lattice [37-41], since both $D^{E}$ and $D_{1}^{E}$ contain perturbative terms $O\left(\frac{1}{x^{4}}\right)$ and $n p$ terms $\exp (-\mu|x|)$, with $\mu \approx O(1 \mathrm{GeV})$, corresponding to the gluelump mass. Finally, one should address the important point of numerical compensation of saturation terms $V_{D}^{\text {sat }}(R)$ and $V_{1}^{\text {sat }}(R)$ in (14) and (17), which otherwise would spoil the linearity of confinement. Indeed both potentials have opposite signs and similar magnitude, and one can easily check, that they compensate each other at the order of $O(10 \%)$, when $M^{(1 g)} \lesssim M^{(2 g)}$.

2. The accuracy of the Casimir scaling in QCD and $\mathrm{SU}(\mathrm{N})$ theory is associated with the magnitude of higher terms $\frac{1}{n !} I_{n}$ in the cluster expansion of $W(C)$, Eq. (2). Indeed, using Eqs. (3) and (6) one can conclude that $\frac{I_{4}}{I_{2}} \lesssim \frac{1}{20}$ and the accuracy of the CS should be around $O(5 \%)$, which is supported by lattice data [21,22], in particular, in [22] the accuracy of CS is around 5\%. One should stress that this result is directly connected to the smallness of $\lambda$, i.e., the large value of the gluelump mass as compared to $\sqrt{\sigma}$. This can be explained as a high stochasticity of the vacuum, where the mean value of the field strength $\langle F\rangle=f$ satisfies the condition $f \lambda^{2} \ll 1$.

At this point one can associate the quantity $f \lambda^{2}$ with the elementary flux on the surface of the Wilson loop and compare it with the corresponding flux of the instanton, $f \lambda^{2}=2 \pi$, while the flux of a magnetic monopole, placed on the surface, is $f \lambda_{m m}^{2}=\pi$ [58], which explains intuitively the range of the magnetic monopole mechanism of confinement, since in this case $W(C)=$ $\exp \sum_{n=1}^{\infty} \frac{\left(i f \lambda_{m m}^{2}\right)^{n}}{n !} \frac{R T_{4}}{\lambda^{2}}=\exp \left(\frac{R T_{4}}{\lambda^{2}} \exp \left(\right.\right.$ if $\left.\left.\lambda^{2}\right)\right) \approx \exp \left(-\frac{R T_{4}}{\lambda_{m m}^{2}}\right)$, $\sigma_{m m} \sim\left(\lambda_{m m}^{2}\right)^{-1} \sim 0.18 \mathrm{GeV}^{2}, \lambda_{m m} \sim 0.5 \mathrm{fm}$.

However, in the FCM case $f \lambda^{2} \sim \sigma \lambda^{2} \sim \frac{\sigma}{\left(M_{0}^{(2 g)}\right)^{2}} \sim O(5 \%)$ and hence one has the picture of stochastic small fluxes in QCD. This picture agrees very well with the lattice measurements in Refs. [21,22].

3. In FCM the flux distributions, given by (24), (25), describe the flux tubes of constant radius which are stabilized at large distance $R$ between charges, as shown in Figs. 6-9, taken from [48]. One can see a good agreement with lattice data of [42], where the distance between charges $R$ belongs to the interval $0.76 \mathrm{fm} \leq R \leq 1.33 \mathrm{fm}$.

In this first step of flux tube theory, given by (24), (25) (see [48] for details), one neglects the perturbative excitations of the string and the width of the flux tube stabilizes at large $R$. In the next order one should take into account an additional gluon in the flux tube, which corresponds to the hybrid state of $(Q G \bar{Q})$. The physics of this static hybrid and the corresponding eigenstates were given in [56] in the framework of FCM. On the lattice these excited states were examined in [54,55].

The formation of the flux tube is often considered in analogy with the Abrikosov fluxes in superconductors and therefore it is one of the main arguments in favor of the DGL picture of confinement in QCD. As a consequence, one tries to find the color magnetic monopole d.o.f. in the QCD vacuum.

However, as shown in $[19,48]$, the flux tube picture occurs naturally in the FCM, as it is demonstrated by Eqs. (24), (25). Indeed, the correlation length $\lambda$ is provided by the gluelump mass $M_{0}^{(2 g)}$, which is created by 
confinement, i.e., by nonzero $\sigma$, and again $\sigma$ is given by the $2 g$ gluelump Green's function $D^{E}(z)$. This mass creation fixed the size of flux tube and its stability, and leads to asymptotic verification of the dual London equation.

One may call it the "microscopic" magnetic monopole mechanism, based on microscopic fluxes $f^{2} \lambda \ll 1$ in contrast to macroscopic DGL mechanism, implying the existence of massive and large magnetic monopole with mass $m_{\chi}$ and dual gauge field with mass $m_{B}$, and their relation $k=\frac{m_{\chi}}{m_{B}}$, which are not predicted by theory. It is very important that in FCM the mass creation process refers to the gluon, connected to another gluon on the given surface of the area law (in the parallel transporter), or to another propagating gluon in the $g g$ glueball, but never refers to one gluon separately. This is important for the problem of color Coulomb screening due to confinement, which is not still observed on the lattice [22] and in the heavy quarkonium spectrum [57]. ${ }^{1}$

Indeed the total potential between static charges both in FCM and on the lattice has the form

$$
V_{\text {static }}^{(j)}(R)=\text { const }_{j}+V_{\text {Coul }}^{(j)}(R)+\sigma^{(j)} R
$$

with

$$
V_{\text {Coul }}^{(j)}(R)=-C_{2}(j) \frac{\alpha_{s}}{R}
$$

with no appreciable screening mass $M_{\text {Coul }}$ and in FCM at zero temperature const ${ }_{j}$ can be put equal to zero. At the same time in the field distribution of the flux tube the Coulomb field contribution to the total color-electric field $\mathbf{E}\left(\frac{\mathbf{R}}{2}\right)$ at the midpoint $\frac{\mathbf{R}}{2}$ between static charges is equal to [48].

$$
\mathbf{E}^{(1)}\left(\frac{\mathbf{R}}{2}\right)=8 C_{2}(j) \frac{\alpha_{s} \mathbf{R}}{R^{3}} \zeta\left(\frac{R}{2 \lambda_{1}}\right), \quad \zeta(x)=(1+x) e^{-x},
$$

which screens with the screening mass $M_{0}^{(1 g)}=1 / 2 \lambda_{1}$, and this behavior is well supported by the lattice data [42].

Thus the FCM theory explains both properties: (1) additivity of color Coulomb and confinement fields in the total $\mathbf{E}$ and (2) the absence of screening in the $V_{\text {Coul }}(R)$, while the screening is present in the flux tube probes.

Indeed, the additivity is based on the additive form of the field correlator (4), which for the total color-electric field $\mathbf{E}$ yields [48]

\footnotetext{
${ }^{1}$ The highly excited states of charmonium and bottomonium may be sensitive to the color Coulomb screening, which strongly decreases the dielectron width [47].
}

$$
E_{i}(\mathbf{r}, \mathbf{R})=n_{k} \int_{0}^{R} d l \int_{-\infty}^{\infty} d t\left(\delta_{i k} D^{E}(z)+\frac{1}{2} \frac{\partial\left(z_{i} D_{i}^{E}(z)\right.}{\partial z_{k}}\right)
$$

where $\mathbf{n}=\mathbf{R} / R$. Calculating (29) with $D_{1}$, given by the one-gluon gluelump Green's function with mass $M_{0}^{(2 g)}=1 / \lambda_{1}$, where one can approximate $\lambda_{1} \approx \lambda$, one obtains the screening factor $\zeta\left(\frac{R}{2 \lambda_{1}}\right)$ for large distances. This refers to the color-electric field, measured as a probe in the Fig. 4.

However, another result obtains if the static potential $V_{\text {Coul }}(R)$ is calculated via $D_{1}^{E}(z)$ (see Appendix B). One can attribute this difference to different Wilson loop constructions in these cases: it is of type of the Fig. 4 for the $\mathbf{E}(\mathbf{r}, \mathbf{R})$ in the probe plaquette and of the Fig. 10 for the $V_{\text {Coul }}(R)$. In last case the screening mass is strongly suppressed due to small change in the area of the covering surface of Wilson loop, lifted by a propagating gluon.

An opposite situation occurs in the interaction of Wilson loops, studied within the FCM theory in [46] and on the lattice in [45]. A particular case of this analysis is the socalled disconnected probe of the field distributions of the static $Q \bar{Q}$ system, which was measured in [12].

Finally, the FCM analysis of flux tubes for three static charges in fundamental and adjoint irreducible representation (irrep) leads to the pictures of 3 quarks with a central string junction in the first case and the triangular configuration in the second case, as shown in Fig. 11 taken from [19], in agreement with lattice and other data.

4. Till now we have discussed the case of static charges, when scalar or vector type of the confining interaction $V_{D}(R)$ is not important. When going to the finite mass quarks, let us consider a light quark in the field of an infinitely heavy antiquark, where the Lorentz nature of $V_{D}(R)$ becomes crucial. Indeed, as shown in $[43,44]$, in the case of the vector confinement the bound state spectrum of the Dirac equation does not exist and it excludes the possibility to use the vector confinement of the GribovZwanziger approach [50] (an exception takes place in the $d=2 \mathrm{QCD}$, if some special transformation of the Bogolyubov-Valatin type is done, see [59]).

The scalar nature of the FCM confining interaction can be directly deduced from the form of $V_{D}$, expressed via $D^{E}(z)$, see Appendix B. Then one can see the two-gluonline exchange form for $V_{D}$ in contrast to the one-gluon-line form for $V_{1}^{E}$, which presupposes the scalar nature for $V_{D}$ and the vector nature for $V_{1}^{E}$ potential. An additional analysis, done in $[60,61]$, supports this conclusion.

5. In all previous analysis we have used the only parameter $\sigma$, while another parameter, $\lambda$, is expressed numerically via $1 / \sqrt{\sigma}, \lambda=\frac{1}{M_{0}^{(2 g)}} \cong \frac{1}{4 \sqrt{\sigma}}$. This situation was checked in $[33,34]$ by calculating the resulting $\alpha_{s}\left(M_{0}^{(2 g)}\right)$ and $\Lambda_{\mathrm{QCD}}$ from the self-consistency equation 
$\sigma_{f}=\frac{1}{2} \int D^{E}(z) d^{2} z$ with $D^{E}(z)$, expressed via $\sigma$ and $\alpha_{s}$ as in (14), (15). In this way one indeed has the only scale in the confinement mechanism [neglecting the quark mass modification of the confinement, which occurs in higher orders of $O\left(g^{n}\right)$ ].

7. The confinement mechanism described above is applicable both to QED and all $S U(N)$ theories, where one explicitly introduces the field correlators $D^{E}(z), D_{1}^{E}(z)$, as in (4). In the QED case, however, as shown in [17], one can apply to the correlator $D_{i \alpha k \beta}(x, y)$ in (4) the operator $\frac{\partial}{\partial x_{\gamma}} \varepsilon_{i \alpha \gamma \delta}$ and take into account the Abelian Bianchi identities, $\partial_{\alpha} \tilde{F}_{\alpha \beta}=0$, which yields $D_{\mathrm{QED}}(z) \equiv 0$, and hence no confinement. One can use the same technique of Gaussian correlators plus gluelump Greens function to other groups, like $G_{2}, F_{4}$ etc. and obtain confinement at intermediate distances, as it was observed on the lattice [26-32,49]. As it was discussed above, a screening of $V_{D}(R)$ due to string breaking may happen in the adjoint loops in $S U(N)$.

\section{THE DGL APPROACH AS THE THEORY OF CONFINEMENT}

The DGL approach, suggested in [2-4] and developed in numerous papers, is reviewed in $[8,10,11]$. In the DGL the original Lagrangian can be written in the Abelian Higgs form (the dual form of it is finally used).

$$
L=-\frac{1}{4} F_{\mu \nu}^{2}-\left|D_{\mu} \varphi\right|^{2}-\frac{\lambda}{4}\left(|\varphi|^{2}-\varphi_{0}^{2}\right), \quad D_{\mu}=\partial_{\mu}-i e A_{\mu}
$$

which obeys the classical solutions-the AbrikosovNielsen-Olesen (ANO) strings, and to describe confinement one needs a region of large $\lambda$, when one has a condensate of electric charges $\varphi=\varphi_{0}$. It is clear, that in this case the field $A_{\mu}$ acquires the mass $m_{B}^{2}=2 e^{2} \varphi_{0}^{2}$, while the Higgs field has its own mass $m_{\chi}^{2}=2 \lambda \varphi_{0}^{2}$. One can easily follow the appearance of London's equations $\Delta \mathbf{B}-m_{0}^{2} \mathbf{B}=0$, implying the stability of magnetic strings with the string tension $\sigma_{\mathrm{ANO}}=\pi m_{B}^{2} \ln \frac{m_{\chi}}{m_{B}}$. Note the possible difficulty in detecting two different mass scales: $m_{\chi}, m_{B}$ where $m_{\chi}$ should be much larger than $m_{B}$ in the proper dual Abrikosov scenario, whereas as we discussed above, one can see on the lattice the only mass scale around $1 \mathrm{GeV}$. Now we consider the properties (1)-(7), presented in the Introduction, with respect to the results of the DGL theory.

(1) The linear behavior of confinement can be ensured by DGL mechanism with a good accuracy (see, e.g., $[8,28]$ and Refs. in [11]) in limited region, which depends on $\lambda \sim \frac{1}{m_{B}}$, and the linear behavior is violated in the region $r \sim \lambda$.

In DGL the behavior of the quadratic field correlators, $G_{2}(x)=\langle F \Phi F\rangle$, as a function of $x$ was investigated in $[10,62]$, with the result: $G_{2}(x)=\frac{c_{1}}{x^{2}} e^{-\mu x}+\frac{c_{2} \exp (-\mu|x|)}{x^{4}}$, which differs from the lattice data [37-41].
(2) The Casimir scaling is strongly violated for fixed values of model parameters $m_{\chi}, m_{B}$ and $k=\frac{m_{\chi}}{m_{B}}$. Indeed, to reach an agreement with the CS values for higher representations $J$ one needs large values of $k \gtrsim 6$ changing with $J[8,25]$.

As we have discussed above in the previous section, the probable reason for this behavior lies in the large values of elementary fluxes $f \lambda^{2}=O(1)$, which bring into action the quartic and higher order correlators, $I_{4} \approx I_{2}$ according to Eq. (6). Large values of $k$ imply the Abrikosov vortex mechanism of the second kind, however, the flux tube profiles require lower $k$ values in the domain of dual superconductors of the first kind [42].

(3) The description of the flux tubes in terms of the DGL theory is rather successful, as shown in [42], however, to reproduce the actual change of the flux tube profile, in [42] it was used the flux tube ansatz [63], based on the type I superconductor model, with three parameters dependent on the charge separation $R$. E.g., the parameter $k$ for $R=0.76 \mathrm{fm}, R=0.95 \mathrm{fm}$, and $R=1.33 \mathrm{fm}$ in Figs. 6-9 should be chosen as $k=0.348,0.170$, and 0.236, respectively.

At the same time, as shown in Figs. 6-9, the FCM produces distributions for all $R$ with the only parameter $\lambda=0.2 \mathrm{fm}$, which is connected to the gluelump mass, calculated via string tension.

One can conclude, that the DGL model corresponds to general picture of flux tubes, at it was expected, however, the microscopic structure of flux tubes is not yet described by a unique theory of the DGL type.

There are also attempts to describe the flux tube as the quantized Nambu-Goto string in the form of the Arvis potential [64] and the induced so-called Lüscher term. However, the latter violates Casimir scaling $[21,22]$. As it is known, the structure of string excitations is associated with an additional gluon d.o.f., as found on the lattice [54,55] and in the FCM [56], while in the standard string theory $[65,66]$ the excitations have different structure.

Summarizing, one can conclude that in the DGL model the flux tube structure is resemblant to that obtained on the lattice for $S U(3)$ theory, but agrees with the latter qualitatively, since there was not found a unique set of parameters describing the lattice data.

(4) It seems natural that in the DGL model one can obtain the scalar confinement, however, corresponding analysis is not known to the author.

(5) The scales and self-consistency of the DGL model was not treated systematically. One clearly defines the scales of dual gauge field $m_{B}=1 / \lambda$ and the dual monopole field $m_{\chi}=1 / \zeta$, and their ratio $k=\frac{m_{\chi}}{m_{B}}$, but their connection to the only $S U(3)$ parameter, $\sigma$, also depends on other parameters, (dual "Higgs" coupling constant $\lambda_{h}$, and its vacuum average $v$ ), so that as in [8], one obtains $\sigma_{f}=4 \pi v^{2}$ in the Bogomol'nyi limit. There are no examples of 
self-consistency checks, where all parameters are deduced from $\sigma$ or $\Lambda_{\mathrm{QCD}}$.

(6) Within the DGL model the Wilson loop-loop or string-string interaction was investigated in the form of the disconnected probes [12]. The point of the color Coulomb nonscreening was not raised in the published literature, to the knowledge of the author.

(7) It is clear that the DGL model can be formulated for any theory, where the gauge d.o.f. are made massive using the dual Higgs field, taken from outside; therefore the main problem is to identify both gauge and dual Higgs d.o.f. This kind of separation and identification is done, e.g., via the Abelian projection method [13], the center vortex model [14], thick vertex model [15], etc. These ideas can be equally well applied to other groups, like it was done in [29] for the groups $G_{2}$ and $S U(N)$. It is difficult to judge whether the DGL explanation is successful unless one derives all parameters directly from the field Lagrangian itself and its renormalization constants. Unfortunately, existing DGL applications are based on the parametrization of given theories in terms of assumed dominant d.o.f. and dominant structure.

\section{THE ABELIAN PROJECTION AND THE CENTER VORTEX MODEL}

One of the most popular version of the confinementconnected studies is the Abelian projection models.

Here one can use the maximally Abelian gauge to separate the field configurations which are believed to contribute maximally to the phenomenon of confinement, see reviews [10,11,14,67]. Ideologically this direction is connected to the idea of monopole dominance, since the necessary gauge transformations might include singular gauge configurations reminiscent of magnetic monopoles. The main idea of the Abelian projection is to extract from the non-Abelian field monopole d.o.f. and to this end one can write in maximally Abelian gauge (MAG) in the $\mathrm{SU}(2)$ case the gauge-transformed plaquette $U_{\mu \nu}$ as $U_{\mu \nu}=$ $\sum_{i} \exp \left(i \theta_{\mu \nu}^{(i)} \sigma_{3}\right)$, with the separation $\theta_{\mu \nu}=\bar{\theta}_{\mu \nu}+2 \pi n_{\mu \nu}$, where $-\pi \lesssim \bar{\theta}_{\mu \nu} \lesssim \pi$ is the Abelian part and $n_{\mu \nu}$ is the "monopole part." The resulting contribution of both parts to the static potential is shown in Fig. 12 [68], where one can see the dominance of the "monopole part" in the string tension and the nonconfining Abelian "photon" contribution. In the lattice studies $[69,70]$ are presented accurate calculations of static quark potential in the maximal Abelian gauge (MAG) in comparison to the exact lattice data for $Q \bar{Q}$ and $3 Q$ configurations. One can see in [69] a very good agreement between MAG and exact values of $\sigma$ with accuracy of the order of 5\%. At the same time the color Coulomb part of the interaction disappears in the MAG version.

How one can understand these results from the point of view of FCM? To this end one must remember that the field correlators $\langle F F\rangle$, responsible for confinement, are produced by the gluelump Green's function, where the color links can be considered as diagonal in the color space in the lowest (nonperturbative) approximation, whereas the color change is provided by the perturbative vertex $g f^{a b c} A_{\mu}^{b} A_{\nu}^{c}$. Hence one can expect that the color diagonalization would keep all the confinement effects up to $O\left(\frac{\alpha_{s}}{\pi}\right)$ and the color exchange potential, generated by perturbative vertices, should be absent in the color diagonal approximation.

In this way the results of the Abelian projection method can be connected to and explained by the FCM.

There appears another question: what are the physical contents of the Abelian projection method (APM)? It is clear that for any physical mechanism of confinement, e.g., for the FCM, the resulting field distributions can be analyzed via the APM, and the only way to explain the confining result is the presence of the $n_{\mu \nu}$ piece in the plaquette, which however has nothing to do with the real monopole admixture, but rather with the fact, that purely Abelian d.o.f. do not ensure confinement, and one needs the "elementary monopole d.o.f." at each point.

The center vortex model (CVM) [14] and its extensionthe thick center vortex model (TCVM) [15]—have attracted a serious attention during last 20 years (see [26-31] and references therein). The main idea of these models, as well as in the DGL approach, is to represent the QCD vacuum as stochastic ensemble of color magnetic fluxes in terms of the nontrivial center elements of the gauge group, and these vortices are thickened in the TCVM, [15] which allowed us to build up the linear potential for all representations at the intermediate distances. As one can see, fundamentally the CVM (TCVM) is based on the notion of the stochasticity of field fluxes connected to center vortices in their total number and orientation. From this point of view there is a similarity between CVM (TCVM) and the FCM approach, since in FCM one has a stochastic ensemble of field fluxes $\hat{F}_{\mu \nu}(z) d \sigma_{\mu \nu}(z)$ inside the Wilson loop, where $\hat{F}_{\mu \nu}(z)$ is the gauge covariant field strength defined in Eq. (2). The stochasticity of the ensemble $\left\{\hat{F}_{\mu \nu}\left(z_{n}\right), n=1,2, \ldots\right\}$ on the plane of the Wilson loop follows from the short correlation length $\lambda=\frac{1}{M_{\mathrm{Glp}}} \lesssim 0.2 \mathrm{fm}$, and as was discussed above [Eq. (6)], the quartic and higher correlators violate the Casimir scaling by less than $5 \%$. Note, that the topological or group structural properties are not necessary for the resulting confinement.

A more detailed group theoretical structure is assigned to the independent field fluxes in CVM (TCVM), where each flux is connected to a center element.

A detailed numerical analysis of the SU(2) Yang-Mills theory in the framework of TCVM was performed in [15], and the linear potentials of static sources have been obtained at intermediate distances. As one can see in Figs. 1, 2 of [15] the linearity of potentials, especially 
for higher representations is indeed achieved in the intermediate region, however there is a problem with the Casimir scaling in this linear region, which is violated for higher representation up to $25 \%$, in contrast to the accurate $(<5 \%)$ scaling in direct lattice calculations.

Another interesting problem in this approach is the significance of the center of group, which can be trivial as in the case of the $G(2)$ group, while $\mathrm{SU}(2)$ and $\mathrm{SU}(3)$ subgroups are present in $G(2)$. This topic was discussed in $[28,31,71]$. It was found in particular in [31] that in $G(2)$ there are two linear regimes at lower $R / a \approx 5$ and $\frac{R}{a} \sim 25$, and for the fist one the Casimir scaling is qualitatively valid.

It is clear, that the FCM method can be directly applied to the simple group theories, e.g., to the $G(2)$ Yang-Mills model and these results can be compared to those in [28] and [31], which can establish a link between the two approaches, in particular it wood be interesting to connect vortex probabilities with the corresponding field correlators.

Here the criterion of the linear confinement between static charges in the $G(2)$ (or any other field theory without quarks) is that all field correlators $\left\langle\hat{F}_{a}\left(x_{1}\right) \hat{F}\left(x_{2}\right) \ldots \hat{F}_{c}\left(x_{n}\right)\right\rangle$ should have exponential asymptotics $\exp \left(-m_{i j}\left|\mathbf{x}_{i}-\mathbf{x}_{j}\right|, \ldots\right)$ with $m_{i j}>0$, and the linear confinement occurs in FCM for $R>1 / m_{i j}$.

As it is, the comparison between the two methods can reveal additional properties of the confinement phenomenon.

\section{SCALAR OR VECTOR CONFINEMENT IN QCD}

Our consideration above has to do with static potentials, where the scalar or vector character of interaction is not important (as will be also seen below). Here we study the case of quarks of any mass and start with the case of a quark of an arbitrary mass $m$ in the field of a static charge. Following $[43,44]$ we write the Dirac Hamiltonian

$$
H=\boldsymbol{\alpha} \mathbf{p}+\beta m+\beta U(r)+V(r), \quad H \psi=E \psi
$$

where $U$ and $V$ are scalar and vector potentials respectively. In the standard bispinor formalism $\Psi=\frac{1}{r}\left(\begin{array}{c}G(r) \Omega \\ i F(r) \Omega^{\prime}\end{array}\right)$, one arrives at the system of equations

$$
\begin{aligned}
& \frac{d G}{d r}+\frac{\kappa}{r} G-(E+m+U-V) F=0 \\
& \frac{d F}{d r}+\frac{\kappa}{r} F+(E-m-U-V) G=0 .
\end{aligned}
$$

Assuming $U=\sigma_{s} r, V=\sigma_{v} r$, we shall consider three possibilities (i) $U \neq 0, V=0$, (ii) $U=0, V \neq 0$, (iii) both $U, V \neq 0$.
Introducing notations $x=\sqrt{\sigma_{s}} r, \varepsilon=E / \sqrt{\sigma_{s}}$, in the case (i) one obtains solutions with the asymptotic

$G, F \sim \exp \left(-\frac{1}{2}\left(x^{2}+b x\right)\right), \quad G^{\prime \prime}-x^{2} G \approx 0$,

implying a reasonable bound state problem even for $m \rightarrow 0$. In the case (ii) replacing $\sigma_{s} \rightarrow \sigma_{v}$, one arrives at the equation

$$
G^{\prime \prime}+x^{2} G=0, \quad G \sim \exp \left(\frac{i x^{2}}{2}\right) .
$$

Thus one cannot have bound states in the vector potential.

In the case (iii) with definition $\sigma_{s}=c_{u} \sigma_{0}, \sigma_{v}=c_{v} \sigma_{0}$, $x=\sqrt{\sigma_{0}} r$, one finally obtains asymptotically at $x \rightarrow \infty$,

$$
G^{\prime \prime}-\left[\left(c_{u} x+\frac{m}{\sqrt{\sigma}}\right)^{2}-c_{v} x^{2}\right] G \approx 0 .
$$

From (35) one can deduce that (1) the necessary condition for the bound state spectrum is $c_{u}>c_{v}$, and (2) for $m \rightarrow \infty$ the bound states exist for any type of confinement.

Comparing this situation with the Gribov-Zwanziger model of confinement [50] one can conclude that the linear vector confinement of this model is not compatible with QCD, unless some additional vacuum transformation of the Bogolyubov-Valatin type is possible, producing finally the scalar confinement, as it happens in the $D=2$ QCD [59].

\section{ADDITIONAL TESTS AND CONSEQUENCES OF THE CONFINEMENT MECHANISM}

When applying the confinement mechanism to real hadron physics, one meets with numerous applications, which serve as a serious test of its nature. Below we shortly discuss several important applications, which should be present in any approach to confinement.

(1) QCD string and Regge trajectories;

(2) confinement in the fast moving hadrons;

(3) the role of confinement in the IR regime and the convergence of the perturbative series;

(4) confinement and chiral symmetry breaking;

(5) confinement and deconfinement at finite temperature. Below we shortly consider all these topics comparing results of confinement mechanisms with real phenomena in hadron physics.

\section{A. QCD string and Regge trajectories}

There are two main approaches to the definition of string spectrum: the first one considers the QCD string, created by confinement, as an example of the string 
theory $[65,66]$ renormalizable in higher dimensions, with its characteristic spectrum of excitations, where each point of string is a dynamical variable and the spectrum is the collective excitation. This type of dynamics was used to calculate contribution of excited string states to the so-called Arvis potential [64] and Nambu-Goto type strings $[65,66]$.

Another approach to the QCD string and the QCD spectrum in general follows from the FCM and can be called the FCM string, where the motion of string is defined by its boundary, i.e., by the ends of the string in the mesons, baryons, and glueballs, $\bar{q} q, 3 q, 3 g$ and will be called configuration $A$; in other case there are extra gluons "string on the string in the excited states", i.e., $\bar{q} g q, \bar{q} g g q, \ldots$ i.e., hybrids, which will be called configuration $B$.

In the FCM string the parts of the string between the ends or internal gluons can be considered as inert and their motion is defined by the endpoints. This is clearly demonstrated by the Nambu-Goto form of the action, exploited in the basic papers of this direction for Regge trajectories [72-74].

Using the relativistic Hamiltonian dynamics [75], one obtains the well-known form of the QCD string Hamiltonian [72-74], describing orbital Regge trajectories of mesons [73], radial meson Regge trajectories [74], Regge trajectories for baryons [76]. There it was demonstrated that this theory works very well for all orbital (proper) Regge trajectories, while for radial Regge trajectories one needs to take into account the flattening of confining potential at large distances-as it is known in QCD with light quarks. The similar trajectories for glueballs are calculated in [77,78], where also the connection with the Pomeron trajectory was studied [78].

The QCD string excitations as hybrid states, introduced in FCM [56], have been compared there with lattice data [54], displaying a reasonable agreement, the same can be said about Regge trajectories in [72-74], where comparison was done with experimental hadron masses. Summarizing this part, one can say that the QCD string picture based on FCM, where the string pieces are treated as inert objects without dynamical d.o.f., is in reasonable agreement with experiment.

\section{B. Confinement in the fast moving hadrons}

It is widely known that in the high-energy collisions fast partons are considered as free particles, not subject to any confinement interaction. This seemingly universal proposal is in contradiction with the relativistically invariant picture, where the transverse d.o.f. can be independent of the (fast) motion of the object.

Indeed, recently in [79] the author has constructed the relativistical invariant Hamiltonian of a fast moving hadron with the resulting solution for the boosted wave function, where the transverse coordinate (momentum) dependence was independent of the motion and coincided with behavior of the wave function in the rest frame. E.g., for the $S$-wave $q \bar{q}$ hadron the boosted wave function can be written as [79]

$$
\varphi\left(p_{\perp}, x\right)=\varphi\left(\sqrt{p_{\perp}^{2}+\left(x_{1}-\frac{1}{2}\right)^{2} M_{0}^{2}}\right),
$$

where $M_{0}$ is the rest mass of the hadron.

As a result, one obtains the valence $p d f$ (the parton distribution function) in the hadron

$$
D_{n}^{q}\left(x, p_{\perp}\right)=\frac{M_{0}^{2}}{(2 \pi)^{3}}\left|\varphi\left(p_{\perp}, x\right)\right|^{2} .
$$

In the total $p d f$ one should take into account all other Fock components, which in the standard formalism [80] are given by free sea quarks etc., while in this "boosted confinement" picture they are represented by fast hadron and hybrid states. The corresponding picture was developed in [81] and is in good agreement with the DIS experiment.

As an additional point the account of the contracted wave functions (36) in the process of $e^{+} e^{-} \rightarrow h h$ allows us to predict the asymptotics of form factors of mesons and baryons (the famous "power law" but without gluon exchanges)

$$
F_{h}\left(Q_{0}\right) \sim\left(\frac{M_{0}^{2}}{Q_{0}^{2}}\right)^{n_{h}}, \quad n_{M}=1, \quad n_{B}=2
$$

where $Q_{0}=\sqrt{M_{0}^{2}+\mathbf{p}^{2}}$.

This topic is of crucial importance for our understanding of high energy (HE) processes and is to be developed further.

In our confinement treatment one can conclude that in the fast moving object the transverse part of confinement is kept intact, so that in all HE collisions, where deconfinement does not occur, all processes are proceeding with confined quarks and gluons in the form of (also) highly excited hadrons, i.e., multihybrids.

Thus confinement strongly affects the standard picture [80] also in the HE process. For considerations, concerning the role of confinement in $\mathrm{HE}$ processes and the $\mathrm{HE}$ momentum sum rule, see [82].

\section{Confinement, IR divergence, and the convergence of perturbative series}

The perturbation series in QCD is subject to serious internal difficulties, as it is known in the textbooks [83], and we will consider those in the following order.

(a) The factorial divergence due to IR renormalons [84].

(b) Landau ghost problem for $\alpha_{s}$ in the IR region [85].

(c) IR divergencies in $\mathrm{QCD}$, being treated as in QED, neglecting confinement [80]. 
(d) The "Euclidean character" of the perturbation theory (PTh) due to absence of hadron thresholds in the analytic dependence $\alpha_{s}(Q)$ etc.

To introduce confinement in the PTh one can use the background perturbation theory (BPTh), as formulated in [86] and developed further in $[87,88]$ with the help of confinement.

As a result one arrives at the PTh, where for all gauge invariant amplitudes, in the higher orders containing closed loops, one takes into account the area law due to confinement. This property strongly changes the behavior at low momenta and, e.g., for the IR renormalon series of amplitudes one arrives at the resulting sum, which does not contain factorial growth at all [89], thus solving the long-standing IR renormalon problem (a).

(b) In a similar way, taking into account the confining loops in the renormalization of $\alpha_{s}$ in the next orders, one arrives at the IR finite form of $\alpha_{s}$ [85], namely, for the one loop one obtains

$$
\alpha_{s}(Q)=\frac{4 \pi}{\beta_{0}} \ln \left(\frac{Q^{2}+M_{B}^{2}}{\Lambda_{\mathrm{QCD}}^{2}}\right),
$$

where $M_{B}^{2}=2 \pi \sigma, M_{B} \cong 1 \mathrm{GeV}$. This result is in a good agreement both with $\alpha_{s}\left(Q^{2}\right)$ for $Q^{2} \gtrsim 3 \mathrm{GeV}^{2}$, as well as with lattice expectations [90-92].

(c) In the HE evolution theory there is a standard agreement that the IR divergence of the QCD amplitudes can be treated similarly to $\mathrm{QED}$, i.e., introducing the gluon mass or the lower limit of the gluon energy $w_{\min }$ to make the divergent amplitudes finite [80].

However confinement precludes the free motion of quark and gluons, placing those on the confining film of the Wilson loop. As a consequence any amplitude of the QCD PTh is IR finite at nonzero $\sigma$ (for examples of IR regularization due to confinement see [93] and Appendix therein). This fact must modify the standard PTh and $p d f$ evolution equations, see [81] for recent results.

(d) The inclusion of confinement in PTh automatically introduces hadron spectra and hadron thresholds $M_{t h}$, and usually in $\alpha_{s}(Q)$ the matching procedure at the current quark masses is used $[80,83]$. Note, that $\sigma$ in $S U(N)$ plays the same role as $\Lambda_{\mathrm{QCD}}$ and one can be expressed via another, as it was shown in [33]. The same role of $\sigma$ retains in QCD with quarks, so that the QCD PTh with confinement contains the same number of RG parameters as the standard PTh, namely $\sigma$ and quark masses $m_{q}$.

\section{Confinement and chiral symmetry breaking}

Confinement and the CSB are internally connected, since the CSB is known only in the systems, where confinement is operating. Moreover, at the growing temperature $T$ the chiral condensate is vanishing just in the region, where the presence of confinement cannot be substantiated.
As was discussed in Sec. V, the resulting confinement for light quarks should be of scalar character (it was argued in $[43,44])$. As a result the presence of the scalar term $\bar{q} M q$ in the effective Lagrangian signals about the CSB. The whole point now is to provide the formalism which ensures both confinement and CSB, and yields all known relations (e.g., GMOR), derived earlier without connection to confinement.

This formalism was created in [94] and generalized in [95], where the effective Lagrangian for quarks with account of confinement and CSB is written in the form

$L_{\text {eff }}=-N_{c} \operatorname{tr} \log (i \hat{\partial}+\hat{m}+M(\mathbf{x}) \hat{U}), \quad \hat{U}=\exp \left(i \hat{\phi} \gamma_{5}\right)$.

Here $\hat{U}$ contains standard Goldstone pseudoscalar mesons, and $M(x) \approx \sigma|\mathbf{x}|$ takes into account confinement of the quark with the antiquark (it is convenient to associate $\mathbf{x}=0$ with the midpoint between $q$ and $\bar{q}$ ). It is also important that at the vertex of the $q \bar{q}$ Green's function, $|\mathbf{x}|=0$ however at $x \lesssim \lambda$ the simple linear behavior is replaced by a more complicated form, to simplify the matter we impose the boundary condition $M(0) \rightarrow M(\lambda)=\sigma \lambda \simeq 0.15 \mathrm{GeV}$.

The Lagrangian (40) allows to obtain all known CSB relations [94,95], where $M(0)$ does not enter, but in addition many new relations, e.g., the effective chiral Lagrangian (ECL) was derived with known coefficients not only in $O\left(p^{2}\right)$ order, but also in $O\left(p^{4}\right), O\left(p^{6}\right)$ [95]. Moreover, the quark coupling constants of $\pi, K--f_{\pi}$, and $f_{K}$ have been calculated in good agreement with lattice and experimental data [96]. The most important point here is that the new chiral-confinement (CC) Lagrangian takes into account both $q, \bar{q}$, and the chiral $(\pi, \pi \pi, K, K \bar{K}, \ldots)$ d.o.f. This is important in the case of external fields, where, e.g., magnetic fields (MF) act directly on the quark d.o.f. and indirectly on the chiral d.o.f. (e.g., on $\pi^{0}, 2 \pi^{0}$..). This important check was done in the MF dependence of quark condensate, $\langle\bar{q} q(B)\rangle$ in [97]. Both results are in good agreement with lattice calculations, which, however, differ from earlier pure chiral d.o.f. results [98].

It is important that in all these cases confinement enters only via string tension $\sigma$ and sometimes via $M(0)=\sigma \lambda$, where $\lambda$ is the same as in the gluelump mass, $\lambda=\frac{1}{M_{\mathrm{glp}}} \approx 0.2 \mathrm{fm}$.

In calculations of the $\pi$ and $K$ masses in MF via $\sigma$ [99], one finds again the dominance of the $q \bar{q}$ over purely chiral d.o.f.

Finally, it is very interesting what happens with CSB with growing quark masses and at which value of $m_{q}$ the chiral properties are lost. This analysis was done in the FCM [100] and compared with lattice and other data. As one can see in [100], the spectra of PS mesons approach the non-CSB form at $m_{q} \gtrsim 150 \mathrm{MeV}$, in agreement with lattice data [101]. 


\section{E. Confinement and deconfinement at growing $T$}

Both $S U(N)$ and QCD with $n_{f}>0$ are subject to the deconfinement process at (or around) some transition temperature $T_{c}$, and at higher $T$ on the lattice one observes the quark-gluon medium, called the quark-gluon plasma (qgp). While in $\mathrm{SU}(3)$ this is the weak first order transition [102], in the case of QCD, $n_{f}=3$ the thermodynamic mechanism is not yet determined. These transitions can be understood qualitatively from the principle of the minimal free energy (maximum pressure), if one neglect artificial Hagedorn states.

The interesting point is that confinement is $T$ dependent and in $\mathrm{SU}(3) \sigma$ decreases before the transition, as shown in [103], which helps to describe well the whole $T$ dependence [102]. The same happens in QCD with $n_{f}=3$, and here the visual phenomenon is the disappearance of the chiral condensate $\langle\bar{q} q(T)\rangle$ at $T \sim 0.16 \mathrm{GeV}$ [104], since in the FCM at small $m_{q},\langle\bar{q} q(T)\rangle \sim(\sigma(T))^{3 / 2}$.

Since FCM is $O(4)$ invariant, it contains both colorelectric (CE) and color-magnetic (CM) correlators, which imply $\mathrm{CE}$ and $\mathrm{CM}$ confinement (CMC). At $T=0$ both $\mathrm{CE}$ and $\mathrm{CM}$ correlators coincide, while with growing $T$ the correlators behave in a different way.

The effect of CMC is dominant for the gluon plasma in $\mathrm{SU}(3)$ and for the $q g p$, as shown in [102,105], where the $\mathrm{CM}$ dynamics was explicitly formulated and the results compared with lattice data. One can expect that CMC is also important for $q \bar{q}$ and $g g$ correlations in the deconfined phase.

The CMC plays an especially important role in the temperature perturbation theory, where it prevents IR divergencies and allows us to solve the Linde problems, by summing a converging infinite set of finite perturbative diagrams [106].

Thus the deconfinement process helps to understand confinement and its structure in detail, and this analysis is becoming more and more informative.

\section{CONCLUSIONS}

The whole discussion above is aimed at the understanding confinement not as a special interesting phenomenon, but rather stressing the point that in QCD confinement is a central property of the whole physical world, which establishes its existence. Indeed, confinement via the baryon masses creates $99 \%$ of the visible energy in the Universe. Therefore all properties of confinement, listed as (1)-(7) in the Introduction and all five consequences in Sec. VII, are intimately connected to each other.

We have shown above that the FCM satisfies all these criteria and is intimately connected to the bilocal field correlator, which provides confinement (the area law of Wilson loops) yielding mass to the gluons inside a hadron. In this respect FCM is similar to the DGL approach, but the FCM mechanism of the gluon mass creation is different.
Indeed, for FCM the quadratic correlator is dominant and this dominance is self-consistent and is supported by the Casimir scaling, while in DGL the confinement mechanism evidently includes higher order correlators. It is interesting to search for the effects, connected to the quartic and higher correlators, possibly associated with the DGL configurations and justifying its presence (at least partial) in the QCD vacuum.

The simplicity of the FCM confinement and its capability to satisfy all criteria, makes it reasonable theory, which can be successfully used in all nine directions, as it was demonstrated above.

With all that, there are still unsolved issues of confinement theory: (1) the mechanism of deconfining temperature transition in full QCD, (2) the temperature variation of field correlators, yielding the decreasing string tension $\sigma(T)$, (3) the role of higher correlators in QCD. The latter can be illustrated by the relation obtained in $[17,57]$

$$
\frac{d D^{E}(z)}{d z^{2}}=\frac{g}{8} f^{a b c}\left\langle F_{\alpha \beta}^{a}(0) F_{\beta \gamma}^{b}(0) F_{\gamma \alpha}^{c}(0)\right\rangle
$$

These topics are presently studied in the framework of the FCM approach.

\section{ACKNOWLEDGMENTS}

The author is grateful to A. M. Badalian for helpful discussions. This work was done in the framework of the scientific project, supported by the Russian Science Foundation, Grant No. 16-12-10414.

\section{APPENDIX A: THE CONTRIBUTION OF THE CORRELATOR $\langle\langle\hat{\boldsymbol{F}} \hat{\boldsymbol{F}} \hat{\boldsymbol{F}} \hat{\boldsymbol{F}}\rangle\rangle$}

The quartic correlator is equal to $\frac{I_{4}}{4 !}$, where

$$
\begin{aligned}
I_{4}= & g^{4} \int_{S} d \sigma(1) \int_{S} d \sigma(2) \int_{S} d \sigma(3) \\
& \left.\times \int_{S} d \sigma(4)\langle\hat{F}(1) \hat{F}(2) \hat{F}(3) \hat{F}(4)\rangle\right\rangle .
\end{aligned}
$$

Since the correlator is self-connected, i.e., it does not depend on position for large area $S$, one can separate one integral, say $\int d \sigma(1)=\operatorname{area}(S)=R T_{4}$, and estimate the rest as follows. The typical construction for the 4- point connected correlator $G(1,2,3,4) \equiv\langle\langle\hat{F}(1) \hat{F}(2) \hat{F}(3) \hat{F}(4)\rangle\rangle$ with $2 g$ vertices $g[A(i) A(i)]$ at each point $i$, is made with $1 g$ propagators connecting neighboring points and the covering film over all construction, so that an upper limit is

$$
\begin{aligned}
G(1,2,3,4) \lesssim & g^{4}\left\langle G^{(1 g)}(1,2) G^{(1 g)}(2,3)\right. \\
& \left.\times G^{(1 g)}(3,4) G^{(1 g)}(4,1)\right\rangle,
\end{aligned}
$$

where $G_{\mu \nu}^{(1 g)}(x, y)=\left\langle\operatorname{Tr}_{a} A_{\mu}(x) \Phi(x, y) A_{\nu}(y)\right\rangle$. Using the asymptotics of $G^{(1 g)}$ in [13] 


$$
\begin{aligned}
G^{(1 g)}(x, y) & \sim\left(N_{c}^{2}-1\right) N_{c} \frac{\sigma_{\text {adj }}}{4 \pi} e^{-M^{(1 g)}|x-y|} \\
& =a e^{-M^{(i g)}|x-y|}
\end{aligned}
$$

one arrives at the estimate

$$
I_{4}=\operatorname{area}(S) \int d^{2} x d^{2} y d^{2} z a^{4} e^{-M^{(1 g)} \sum_{i j} r_{i j}}
$$

with $a=\frac{g N_{c}\left(n_{c}^{2}-1\right) \sigma_{\mathrm{adj}}}{4 \pi}$.

The integral yields the factor $\frac{\text { const }}{\left(M^{1 g}\right)^{6}}$, with const $\approx O(1)$, so that the final estimate is

$$
\begin{aligned}
I_{4} & =\operatorname{area}(S)\left(\frac{g N_{c}\left(n_{c}^{2}-1\right)}{4 \pi}\right)^{4} \sigma_{\text {adj }} \frac{\sigma_{\text {adj }}^{3}}{\left(M^{(1 g)}\right)} \\
& \approx \sigma_{\text {adj }} \operatorname{area}(S)\left(\sigma \lambda^{2}\right)^{3} .
\end{aligned}
$$

Since $\sigma \lambda^{2} \approx 0.05$, one can see a strong suppression factor, ensuring the Casimir scaling at the order $O\left(F^{4}\right)$.

\section{APPENDIX B}

We start with the standard definition of quadratic correlator [13]

$$
\begin{aligned}
D_{\mu \nu, \lambda \sigma}(x, 0)= & \frac{g^{2}}{N_{c}}\left\langle\operatorname{tr} F_{\mu \nu}(x) \Phi(x, 0) F_{\lambda \sigma}(0)\right\rangle \\
= & \left(\delta_{\mu \lambda} \delta_{\nu \sigma}-\delta_{\mu \sigma} \delta_{\nu \lambda}\right) D(x) \\
& +\frac{1}{2}\left[\frac{\partial}{\partial x_{\mu}}\left(x_{\lambda} \delta_{\nu \sigma}-x_{\sigma} \delta_{\nu \lambda}\right)+(\mu \lambda \rightarrow \nu \sigma)\right] D_{1}(x) .
\end{aligned}
$$

To express static potential $V(R)$ via $F_{i 4}$ one can use the representation

$$
W\left(R, T_{4}\right)=\exp \left(-\frac{g^{2}}{2} \int\langle F \Phi F\rangle d \sigma d \sigma^{\prime}\right)=\exp \left(-\hat{V} d t_{4}\right)
$$

and express the correlator $\langle F(x) F(y)\rangle \sim D$ as in (B1). As a result one obtains

$$
\begin{aligned}
\int \hat{V} d t_{4}= & \frac{1}{2} \int D_{14,14}(u-v) d^{2} u d^{2} v \\
= & \frac{1}{2} \int d\left(\frac{u_{4}+v_{4}}{2}\right) d\left(u_{4}-v_{4}\right) d\left(\frac{u_{1}+v_{1}}{2}\right) \\
& \times d\left(u_{1}-v_{1}\right) D_{14,14}(u-v) \\
= & 2 \int d t_{4} \int_{0}^{\infty} d \nu \int_{0}^{R} d \eta(R-\eta)(D(\nu, \eta) \\
& +\frac{1}{2} \frac{d}{d \eta}\left(\eta D_{1}(\nu, \eta)\right)
\end{aligned}
$$

where $t_{4}=\frac{u_{4}+v_{4}}{2}, \nu=\left|u_{4}-v_{4}\right|, \eta=\left|u_{1}-v_{1}\right|$, one finally obtains

$$
\begin{aligned}
V & =V_{D}(R)+V_{1}(R), \\
V_{D} & =2 \int_{0}^{\infty} d \nu \int_{0}^{R} d \eta(R-\eta) D(\nu, \eta) \\
V_{D}(R) & =V_{\text {lin }}(R)+V_{D}^{(\text {sat })}(R)
\end{aligned}
$$

and consider the color-electric correlator $D_{1}^{E}(x)$ putting $\nu=\sigma=4$, which produces the potential $V_{1}(r)$.

$$
V_{1}(r)=\int_{0}^{r} \lambda d \lambda \int_{0}^{\infty} d \tau D_{1}^{E}\left(\sqrt{\lambda^{2}+\tau^{2}}\right)
$$

As a new step one must express $D_{1}(x)$ via the gluelump Green's function to the lowest order in background perturbation theory. To this end one can extract from $F_{\mu \nu}(x)=\partial_{\mu} A_{\nu}-\partial_{\mu} A_{\mu}-i g\left[A_{\mu}, A_{\nu}\right]$, the part with derivatives, which contributes to $D_{1}$

$$
\begin{aligned}
& D_{1 \mu \nu, \lambda \sigma}^{(0)}(x, y) \\
& =\frac{g^{2}}{2 N_{c}^{2}}\left\{\frac{\partial}{\partial x_{\mu}} \frac{\partial}{\partial y_{\lambda}}\left\langle\operatorname{tr}_{a} A_{\nu}(x) \Phi(x, y) A_{\sigma}(y)\right\rangle+\text { perm }\right\}
\end{aligned}
$$

and denoting as $G^{(1 g)}$ the structure in the angular brackets, one obtains for $\mu=\lambda=4$.

$$
\frac{\partial}{\partial x_{4}} \frac{\partial}{\partial y_{4}} G_{\nu \sigma}^{(1 g)}(x-y)=\frac{\partial}{\partial x_{4}}\left(x_{4}-y_{4}\right) D_{1}(x-y) \delta_{\nu \sigma}+\text { perm } .
$$

As a result one obtains that $G_{\nu \sigma}^{(1 g)}(z)=\delta_{\nu \sigma} G^{(1 g)}(z)$ and $D_{1}^{E}$ is connected to $G^{(1 g)}$ as

$$
D_{1}^{E}(x)=-\frac{2 g^{2}}{N_{c}^{2}} \frac{d G^{(1 g)}}{d x^{2}} .
$$

Here $G^{(1 g)}(x-y)$ is the Green's function of the gluon gluelump, i.e., the gauge invariant combination of the gluon propagator augmented with the parallel transporter $\Phi(x, y)$, as shown in Fig. 1.

This function and the corresponding gluelump spectrum was found analytically [35], being in a good agreement with the lattice data [36].

Inserting (3) into (2), one obtains the relation between $V_{1}(r)$ and $G^{(1 g)}$, namely

$$
V_{1}(r)=-\frac{g^{2}}{N_{c}^{2}} \int_{0}^{\infty} d \tau\left(G^{(1 g)}\left(\sqrt{r^{2}+\tau^{2}}\right)-G^{(1 g)}(\tau)\right) .
$$

Since $G^{(1 g)}(x \rightarrow \infty) \rightarrow 0$ one obtains 


$$
\begin{aligned}
V_{1}(\infty) & =\frac{g^{2}}{N_{c}^{2}} \int_{0}^{\infty} d \tau G^{(1 g)}(\tau) . \\
V_{1}(r) & =V_{\text {coul }}+V_{1}^{(\text {sat })}
\end{aligned}
$$

Therefore to define properly the perturbative Coulomb interaction one can write,

$$
\begin{aligned}
V_{1}^{(\text {pert })}(r) & =\frac{8 \alpha_{s}}{3 \pi} \int_{0}^{\infty} d \nu\left(\frac{1}{\nu^{2}}-\frac{1}{\nu^{2}+r^{2}}\right) \\
& =V_{1}^{(\text {pert })}(\infty)-\frac{4 \alpha_{s}}{3 r}
\end{aligned}
$$

and to renormalize (24) one can put $V_{1}^{(\text {pert })}(\infty)=0$.

In a similar way one introduces the two-gluon gluelump, as it was done in [33-35]

$$
\begin{aligned}
D_{i k l m}(x, y) & =D_{i k, l m}^{(0)}+D_{i k, l m}^{(1)}+D_{i k, l m}^{(2)} . \\
D_{i k, l m}^{(2)}(x, y) & =-\frac{g^{4}}{2 N_{c}^{2}}\left\langle\operatorname{tr}_{a}\left(\left[a_{i}, a_{k}\right] \hat{\Phi}(x, y)\left[a_{l}, a_{m}\right]\right)\right\rangle . \\
{\left[a_{i}, a_{k}\right] } & =i a_{i}^{a} a_{k}^{b} f^{a b c} T^{c} \\
G_{i k, l m} & =\operatorname{tr}_{a}\left\langle f^{a b c} f^{d e f} a_{i}^{a}(x) a_{k}^{b}(x) T^{c} \hat{\Phi}(x, y) T^{f} a_{l}^{d} a_{m}^{e}\right\rangle .
\end{aligned}
$$

$G_{i k, l m}(x, y)=N_{c}^{2}\left(N_{c}^{2}-1\right)\left(\delta_{i l} \delta_{\mathrm{km}}-\delta_{i m} \delta_{k l}\right) G^{(2 \mathrm{gl})}(x, y)$,

$$
\begin{gathered}
D(x-y)=\frac{g^{4}\left(N_{c}^{2}-1\right)}{2} G^{(2 \mathrm{gl})}(x, y) . \\
G^{(2 \mathrm{gl})(0)}(x, y)=\frac{1}{\left(4 \pi^{2}(x-y)^{2}\right)^{2}}+O \frac{\left(\alpha_{s} \ln (x-y)\right)}{(x-y)^{4}},
\end{gathered}
$$

$$
\sigma=\frac{1}{2} \int d^{2} x(D(x)+\text { higher correlators })
$$

$$
G^{(2 \mathrm{gl})}(T)=\sum\left|\Psi_{n}^{(2 \mathrm{gl})}(0)\right|^{2} e^{-M_{n}^{(2 \mathrm{gl})} T} .
$$

\section{APPENDIX C: SELF-CONSISTENCY OF $\sigma_{f}$}

The behavior of $G^{(2 g)}(z), D^{E}(z)$ for $z \rightarrow 0$ was analyzed in $[33,34]$, where it was shown that at $z \ll \lambda, D_{n p}^{E}(z) \equiv$ $D^{E}(z)$, after subtraction of the divergent term, $G^{2 g)}(z)=\frac{\text { const }}{z^{4}}$

$$
\begin{aligned}
G^{2 g}(z) & =G_{\text {pert }}^{2 g)}(z)+G_{n p}^{2 g)}(z) \\
G_{\text {pert }}^{2 g)}(z) & =\frac{1}{\left(4 \pi^{2} z^{2}\right)^{2}}+O\left(\frac{\alpha_{s} \ln z}{z^{4}}\right)
\end{aligned}
$$

has a minimum at $z=0$, namely

$$
D^{E}(0) \cong \frac{N_{c}}{2 \pi^{2}}\left(\frac{2 \pi}{\beta_{0}}\right)^{2} D^{E}(\lambda)
$$

which however does not change appreciably the integral (5), so that one can write

$$
\sigma_{f}=\frac{1}{2} \int d^{2} z D^{E}(z), \quad D^{E}(z) \cong a e^{-M|z|}, \quad M \equiv M_{0}^{(2 g)},
$$

where $a$ according to (15) is

$$
a=16 \pi^{2} \alpha_{s}^{2}(M) \cdot 0.4 \sigma_{f}^{2} .
$$

From $(\mathrm{C} 1)$ and $(\mathrm{C} 2)$ one obtains the self-consistency condition

$$
\sigma_{f} \geq 16 \pi^{2} \cdot 0.4 \sigma_{f}^{2} \alpha_{s}^{2}(M) \frac{\pi}{M^{2}},
$$

where one should take into account the relation

$$
\alpha_{s}(M)=\frac{4 \pi}{\beta_{0} \ln \left(\frac{M^{2}+M_{B}^{2}}{\Lambda^{2}}\right)}
$$

with $M_{B} \cong \sqrt{2 \pi \sigma_{f}} \approx 1 \mathrm{GeV}, M=2 \mathrm{GeV}$, where we take into account the IR regularization of $\alpha_{s}$ [85]. As a result one obtains from (C3) and (C4) the connection of $\Lambda_{\sigma}$ and $\sigma$

$\Lambda_{\sigma}^{2}=\left(M^{2}+M_{B}^{2}\right) \exp \left(-\frac{\sqrt{\sigma_{f}}}{M} 17.7 \frac{\sqrt{1-1 / N_{c}^{2}}}{\left(1-\frac{2}{11} \frac{n_{f}}{N_{c}}\right)}\right)$,

and for $\sigma_{f}=0.18 \mathrm{GeV}, M=2 \mathrm{GeV}, M_{B}=1 \mathrm{GeV}, N_{c} \rightarrow \infty$ one obtains $\Lambda_{\sigma}=0.342 \mathrm{GeV}$. Here one must take into account the difference between the space constant $\Lambda_{\sigma}$ and the momentum space $\Lambda_{\mathrm{QCD}}$, see $[91,92]$ for the analysis, $\Lambda_{\sigma} \approx 1.3 \Lambda_{\mathrm{QCD}}$, yielding $\Lambda_{\mathrm{QCD}}=0.29 \mathrm{GeV}$.

One can check that for $\sigma=0.18 \mathrm{GeV}^{2}, M=2 \mathrm{GeV}$ one obtains $\alpha_{s}(2 \mathrm{GeV}) \approx 0.33$, which is within the PDG limits, and both relations $(\mathrm{C} 3)$ and $(\mathrm{C} 4)$ are satisfied. In this way the self-consistency check shows the reliability of the obtained gluelump string tension.

In addition one check the behavior of $D^{E}(z)$ in (C1) vs lattice data for $D^{E}(z)$ and $D_{1}^{E}(z)$, obtained in [37-41].

The general structure of these correlators on the lattice can be approximated as $D^{E} \sim \frac{a}{z^{4}}+b e^{-z / \lambda}$, with $\lambda \sim 0.2 \mathrm{fm}$ in reasonable agreement with $(\mathrm{C} 1)$. As it is discussed in Sec. IV, this lattice behavior contradicts the field correlators obtained in DGL, $D^{E}, D_{1}^{E} \sim O\left(\frac{e^{-z / \lambda}}{z^{4}}\right)$, see [10,62] for more detail. 
[1] K. G. Wilson, Phys. Rev. D 10, 2445 (1974).

[2] Y. Nambu, Phys. Rev. D 10, 4262 (1974).

[3] G.'t Hooft, in High Energy Physics, edited by A. Zichichi (Editrice Compositori, Bologna, 1976).

[4] S. Mandelstam, Phys. Lett. 53B, 476 (1975); Phys. Rep. 23, 245 (1976).

[5] A. M. Polyakov, Nucl. Phys. B120, 429 (1977); Phys. Lett. 59B, 80 (1975).

[6] A. A. Abrikosov, Sov. Phys. JETP 5, 1174 (1957).

[7] H. B. Nielsen and P. Olesen, Nucl. Phys. B61, 45 (1973).

[8] Y. Koma, E. M. Ilgenfritz, H. Toki, and T. Suzuki, Phys. Rev. D 68, 114504 (2003); M. Baker, J. S. Ball, and F. Zakharisen, Phys. Rev. D 41, 2612 (1990); Phys. Rep. 209 , 73 (1991).

[9] T. Suzuki, Prog. Theor. Phys. 80, 929 (1988); S. Maedan and T. Suzuki, Prog. Theor. Phys. 81, 229 (1989).

[10] G. Ripka, Lect. Notes Phys. 639, 1 (2004).

[11] K. I. Kondo, S. Kato, A. Shibata, and T. Shinohara, Phys. Rep. 579, 1 (2015).

[12] M. Fukugita and T. Niuya, Phys. Lett. 132B, 374 (1983); J. E. Kiskis and K. Sparks, Phys. Rev. D 30, 1326 (1984); J. W. Flower and S. W. Otto, Phys. Lett. 160B, 128 (1985); J. Wosiek and R. W. Haymaker, Phys. Rev. D 36, 3297 (1987); A. Di Giacomo, M. Maggiore, and S. Olejnik, Phys. Lett. B 236, 199 (1990); A. Di Giacomo, M. Maggiore, and S. Olejnik, Nucl. Phys. B347, 441 (1990); V. Singh, D. A. Browne, and R. W. Haymaker, Phys. Lett. B 306, 115 (1993); P. Cea and L. Cosmai, Nucl. Phys. B, Proc. Suppl. 30, 572 (1993); Y. Matsubara, S. Ejiri, and T. Suzuki, Nucl. Phys. B, Proc. Suppl. 34, 176 (1994); G. S. Bali, K. Schilling, and C. Schlichter, Phys. Rev. D 51, 5165 (1995); T. T. Takahashi, H. Suganuma, Y. Nemofo, and H. Matsufuru, Phys. Rev. D 65, 114509 (2009); R. W. Haymaker and T. Matsuki, Phys. Rev. D 75, 014501 (2007); A. D'Alessandro, M. D'Elia, and L. Tagliacozzo, Nucl. Phys. B774, 168 (2007); M. S. Cardaci, P. Cea, L. Cosmai, R. Falcone, and A. Papa, Phys. Rev. D 83, 014502 (2011); P. Cea, L. Cosmai, and A. Papa, Phys. Rev. D 86, 054501 (2012); N. Cardoso, M. Cardoso, and P. Bicudo, Phys. Rev. D 88, 054504 (2013); M. Caselle, M. Panero, R. Pellegrini, and D. Vadacchino, J. High Energy Phys. 01 (2015) 105.

[13] G.'t Hooft, Nucl. Phys. B190, 455 (1981).

[14] G.'t Hooft, Nucl. Phys. B138, 1 (1978); J. Greensite, Prog. Part. Nucl. Phys. 51, 1 (2003).

[15] M. Faber, J. Greensite, and S. Olejnik, Phys. Rev. 57, 2603 (1998).

[16] H. G. Dosch and Yu. A. Simonov, Phys. Lett. B 205, 339 (1988).

[17] Yu. A. Simonov, Phys. Usp. 166, 337 (1996).

[18] A. Di Giacomo, H. G. Dosch, V. I. Shevchenko, and Yu. A. Simonov, Phys. Rep. 372, 319 (2002).

[19] D. S. Kuzmenko, V. I. Shevchenko, and Yu. A. Simonov, Phys. Usp. 47, 1 (2004).

[20] N. Campbell, I. Jorysz, and C. Michael, Phys. Lett. B 167, 91 (1986); C. Michael, Nucl. Phys. B, Proc. Suppl. 26, 417 (1992).

[21] S. Deldar, Phys. Rev. D 62, 034509 (2000).

[22] G. Bali, Phys. Rev. D 62, 114503 (2000).
[23] V. I. Shevchenko and Yu. A. Simonov, Phys. Rev. Lett. 85, 1811 (2000).

[24] V. I. Shevchenko and Yu. A. Simonov, arXiv:hep-ph/ 0104135 .

[25] Y. Koma and M. Koma, Eur Phys. J. C 26, 457 (2003).

[26] L. Liptak and S. Olejnik, Phys. Rev. D 78, 074501 (2008).

[27] B. H. Wellegehausen, A. Wipf, and C. Wozar, Phys. Rev. D 83, 114502 (2011); 83, 01600 (2011).

[28] J. Greensite, K. Langfeld, S. Olejnik, H. Reihardt, and T. Toki, Phys. Rev. D 75, 034501 (2007).

[29] S. Deldar, H. Lookzadeh, and S. M. H. Nejad, Phys. Rev. D 85, 054501 (2012).

[30] S. Deldar and S. M. H. Nejad, Phys. Rev. D 89, 014510 (2014).

[31] S. M. H. Nejad and S. Deldar, J. High Energy Phys. 03 (2015) 016.

[32] A. Shahlaei and S. Rafibakhsh, Phys. Rev. D 97, 056015 (2018).

[33] Yu. A. Simonov, Phys. At. Nucl. 69, 528 (2006); Proc Steklov Inst Math / Trudy Matematicheskogo instituta imeni VA Steklova 272, 234 (2011).

[34] Yu. A. Simonov and V. I. Shevchenko, Adv. High Energy Phys. 2009, 1 (2009).

[35] Yu. A. Simonov, Nucl. Phys. B592, 350 (2001).

[36] I. Jorysz and C. Michael, Nucl. Phys. B302, 448 (1988); N. Campbell, I. Jorysz, and C. Michael, Phys. Lett. 167B, 91 (1986).

[37] A. Di Giacomo and H. Panagopoulos, Phys. Lett. B 285, 133 (1992).

[38] M. D’Elia, A. Di Giacomo, and E. Meggiolaro, Phys. Lett. B 408, 3151 (1997).

[39] A. Di Giacomo, E. Meggiolaro, and H. Panagopoulos, Nucl. Phys. B483, 371 (1997).

[40] M. D’Elia, A. Di Giacomo, and E. Meggiolaro, Phys. Rev. D 67, 114504 (2003).

[41] G. S. Bali, N. Brambilla, and A. Vairo, Phys. Lett. B 421, 265 (1998).

[42] P. Cea, L. Cosmai, F. Cuteri, and A. Papa, Phys. Rev. D 95, 114511 (2017).

[43] V. D. Mur, V. S. Popov, Yu. A. Simonov, and V. P. Yurov, J. Exp. Theor. Phys. 78, 1 (1994).

[44] Yu. A. Simonov, JETP Lett. 106, 135 (2017).

[45] V. G. Bornyakov, P. Yu. Boyko, M. N. Chernodub, and M. I. Polikarpov, arXiv:hep-lat/0508006.

[46] I. Shevchenko and Yu. A. Simonov, Phys. Rev. D 66, 056012 (2002).

[47] A. M. Badalian and B. L. Bakker, Phys. Rev. D 93, 074034 (2016).

[48] M. S. Lukashov and Yu. A. Simonov, Phys. Rev. D 96, 076019 (2017).

[49] K. Holland, P. Minkowski, M. Pepe, and U. J. Wiese, Nucl. Phys. B668, 207 (2003).

[50] V. N. Gribov, Nucl. Phys. B139, 1 (1978); D. Zwanziger, Nucl. Phys. B518, 237 (1998); A. Cucchieri and D. Zwanziger, Phys. Rev. Lett. 78, 3814 (1997).

[51] V. Volterra and B. Hostinsky, Operations Infinitesimal e Lineaires (Gauthiers Villars, Paris, 1939); M. B. Halpern, Phys. Rev. D 19, 517 (1979); N. Bralic, Phys. Rev. D 22, 3090 (1980); I. Arefeva, Theor. Math. Phys. 43, 353 (1980); Yu. A. Simonov, Phys. At. Nucl. 50, 213 (1989). 
[52] N. G. Van Kampen, Physica (Amsterdam) 74, 215 (1974); Phys. Rep. 24C, 171 (1976).

[53] Yu. A. Simonov, Phys. At. Nucl. 66, 338 (2003).

[54] C. Michael, Nucl. Phys. A655, c12 (1999); G. Bali, G. Bolder, N. Eicker, Thomas Lippert, Boris Orth, Peer Ueberholz, Klaus Schilling, and Thorsten Struckmann, Phys. Rev. D 62, 054503 (2000); K. J. Juge, J. Kuti, and C. Morningstar, Phys. Rev. Lett. 90, 161601 (2003).

[55] G. Bali and A. Pineda, Phys. Rev. D 69, 094001 (2004); C. Reisinger, S. Capitani, O. Philipsen, and M. Wagner, arXiv:1708.05562.

[56] Yu. A. Simonov, Nucl. Phys. B, Proc. Suppl. 23, 283 (1991); Yu. S. Kalashnikova and A. V. Nefediev, Phys. Rev. D 77, 054025 (2008); Yu. S. Kalashnikova and D. S. Kuzmenko, Phys. At. Nucl. 66, 955 (2003); Yu. S. Kalashnikova and Yu. B. Yufryakov, Phys. Lett. B 359, 175 (1995).

[57] G. S. Bali, Int. J. Mod. Phys. A 21, 5610 (2006).

[58] Yu. A. Simonov, Sov. J. Nucl. Phys. 50, 134 (1989).

[59] Yu. S. Kalashnikova and A. V. Nefediev, Phys. Usp. 45, 347 (2002).

[60] Yu. A. Simonov, Phys. At. Nucl. 60, 2069 (1997).

[61] A. V. Nefediev and Yu. A. Simonov, JETP Lett. 82, 557 (2005).

[62] H. G. Dosch, M. Baker, N. Brambilla, and A. Vairo, Phys. Rev. D 58, 034010 (1998).

[63] J. R. Clem, J. Low Temp. Phys. 18, 427 (1975).

[64] J. Arvis, Phys. Lett. 127B, 106 (1983).

[65] S. Daley, Phys. Rev. D 74, 014025 (2006).

[66] Y. Nambu, Phys. Rev. D 10, 4262 (1974); T. Goto, Prog. Theor. Phys. 46, 1560 (1971); J. Maldacena, Adj. Theor. Math. Phys. 2, 231 (1998); A. M. Polyakov, Int. J. Mod. Phys. A 14, 645 (1999).

[67] M. N. Chernodub and M. I. Polikarpov, in Confinement, Duality, and Nonperturbative Aspects of QCD. Proceedings, NATO Advanced Study Institute, Newton Institute Workshop, Cambridge, UK, 1997 (Plenum, New York, 1998), pp. 387-414.

[68] G. S. Bali, V. G. Bornyakov, M. Müller-Preussker, and K. Schilling, Phys. Rev. D 54, 2863 (1996).

[69] H. Suganuma and N. Sakumichi, arXiv:1812.06827.

[70] N. Sakumichi and H. Suganuma, Phys. Rev. D 90, 111501 (2014); 92, 034511 (2015).

[71] J. Greensite and K. Matsuyama, Phys. Rev. D 96, 094510 (2017).

[72] A. Yu. Dubin, A. B. Kaidalov, and Yu. A. Simonov, Phys. Lett. B 323, 41 (1994).

[73] A. M. Badalian and B. L. G. Bakker, Phys. Rev. D 66, 034025 (2002).

[74] A. M. Badalian, B. L. G. Bakker, and Yu. A. Simonov, Phys. Rev. D 66, 116004 (2002).

[75] Yu. A. Simonov, Nucl. Phys. B307, 512 (1988); Yu. A. Simonov and J. A. Tjon, Ann. Phys. (N.Y.) 300, 54 (2002); Yu. A. Simonov, Phys. Rev. D 88, 025028 (2013).

[76] M. Fabre dela Ripelle and Yu. A. Simonov, Ann. Phys. (N.Y.) 212, 235 (1991).

[77] A. B. Kaidalov and Yu. A. Simonov, Phys. Lett. B 477, 163 (2000); Phys. At. Nucl. 63, 1428 (2000).
[78] A. B. Kaidalov and Yu. A. Simonov, Phys. Lett. B 636, 101 (2006).

[79] Yu. A. Simonov, Phys. Rev. D 91, 065001 (2015).

[80] R. P. Feynman, Photon-Hadron Interactions (W.A. Benjamin Inc., New York, 1972); B. L. Ioffe, V. A. Khose, and L. N. Lipatov, Deep Inelastic Processes (North-Holland, Amsterdam, 1984); F. J. Yndurain, The Theory of Quark and Gluon Interactions (Springer, New York, 2006).

[81] Yu. A. Simonov, Phys. At. Nucl. 79, 419 (2016).

[82] J. C. Collins, D. E. Soper, and G. Sterman, Nucl. Phys. B261, 104 (1985).

[83] B. L. Ioffe, V. S. Fadin, and L. N. Lipatov, Quantum Chromodynamics (Cambridge University Press, Cambridge, England, 2010).

[84] G.'tHooft, in The Whys of Subnuclear Physics, edited by A. Zichichi (Plenum, New York and London, 1977), p. 74.

[85] Yu. A. Simonov, Phys. At. Nucl. 74, 1223 (2011).

[86] B. S. De Witt, Phys. Rev. 162, 1195, 1239 (1967); J. Honerkamp, Nucl. Phys. B48, 269 (1972); G. t Hooft, Nucl. Phys. B62, 44 (1973); Acta Univ. Wratislaviensis 368, 345 (1976); L. F. Abbot, Nucl. Phys. 185, 189 (1981).

[87] Yu. A. Simonov, in Lecture Notes in Physics (SpringerVerlag, Berlin, 1996), Vol. 479, p. 139; Yad. Fiz. 58, 113 (1995).

[88] Yu. A. Simonov, Phys. At. Nucl. 65, 135 (2002).

[89] Yu. A. Simonov, JETP Lett. 57, 525 (1993).

[90] G. S. Bali, Phys. Lett. B 460, 170 (1999).

[91] A. M. Badalian, Phys. At. Nucl. 63, 2173 (2000).

[92] A. M. Badalian and D. S. Kuzmenko, Phys. Rev. D 65, 016004 (2001).

[93] Yu. A. Simonov, Phys. Rev. D 96, 096002 (2017).

[94] Yu. A. Simonov, Phys. Rev. D 65, 094018 (2002); Phys. At. Nucl. 67, 846 (2004); 67, 1027 (2004).

[95] Yu. A. Simonov, Int. J. Mod. Phys. A 31, 165016 (2016).

[96] Yu. A. Simonov, Phys. At. Nucl. 79, 265 (2016).

[97] V. D. Orlovsky and Yu. A. Simonov, J. High Energy Phys. 01 (2014) 118.

[98] I. A. Shushpanov and A. V. Smilga, Phys. Lett. B 402, 351 (1997).

[99] V. D. Orlovsky and Yu. A. Simonov, J. High Energy Phys. 09 (2013) 135.

[100] Yu. A. Simonov, Phys. At. Nucl. 76, 525 (2013).

[101] D. Galletly, M. Gürtler, R. Horsley, H. Perlt, P. E. L. Rakow, G. Schierholz, A. Schiller, and T. Streuer, Phys. Rev. D 75, 073015 (2007).

[102] N. O. Agasian, M. S. Lukashov, and Yu. A. Simonov, Eur. Phys. J. A 53, 138 (2017).

[103] O. Kaczmarek, F. Karsch, E. Laermann, and M. Lutgerneier, Phys. Rev. D 62, 034021 (2000).

[104] A. Bazavov, T. Bhattacharya, G. De Tar et al., Phys. Rev. 90, 094503 (2014).

[105] M. A. Andreichikov, M. S. Lukashov, and Yu. A. Simonov, Int. J. Mod. Phys. A 33, 1850043 (2018).

[106] Yu. A. Simonov, Phys. Rev. D 96, 096002 (2017). 